\title{
Working memory, perceptual priming, and the perception of hierarchical forms: Opposite effects of priming and working memory without memory refreshing
}

\author{
JEONG-IM KIM AND GLYN W. HuMPhREYS \\ University of Birmingham, Birmingham, England
}

\begin{abstract}
Previous research has shown that stimuli held in working memory (WM) can influence spatial attention. Using Navon stimuli, we explored whether and how items in WM affect the perception of visual targets at local and global levels in compound letters. Participants looked for a target letter presented at a local or global level while holding a regular block letter as a memory item. An effect of holding the target's identity in WM was found. When memory items and targets were the same, performance was better than in a neutral condition when the memory item did not appear in the hierarchical letter (a benefit from valid cuing). When the memory item matched the distractor in the hierarchical stimulus, performance was worse than in the neutral baseline (a cost on invalid trials). These effects were greatest when the WM cue matched the global level of the hierarchical stimulus, suggesting that WM biases attention to the global level of form. Interestingly, in a no-memory priming condition, target perception was faster in the invalid condition than in the neutral baseline, reversing the effect in the WM condition. A further control experiment ruled out the effects of WM being due to participants' refreshing their memory from the hierarchical stimulus display. The data show that information in WM biases the selection of hierarchical forms, whereas priming does not. Priming alters the perceptual processing of repeated stimuli without biasing attention.
\end{abstract}

Many everyday tasks require that we select from the environment stimuli relevant to our behavioral goals. Normally, this process of goal-directed selection may depend on a match taking place between goal-related information in working memory (WM) and incoming stimulus information (Desimone \& Duncan, 1995; Duncan, 1998). Evidence supporting the role of top-down guidance of search to targets comes from experiments showing that search benefits by providing participants with foreknowledge of targets (e.g., Anderson, Heinke, \& Humphreys, in press; Wolfe, 2005), and that indeed some targets only "pop out" when foreknowledge is given (Hodsoll \& Humphreys, 2001). Recent work suggests that these top-down effects are not confined to holding knowledge of the target, since there can also be effects of irrelevant information in WM. Downing (2000) had participants hold one stimulus in WM, then search for another target. The irrelevant cue in WM could reappear alongside the target or another item. Response times (RTs) were faster when the WM stimulus reappeared at the target's location (on valid trials) than when it fell elsewhere (on invalid trials). Importantly, this effect did not occur when the cue was presented but did not have to be maintained in memory. Soto, Heinke, Humphreys, and Blanco (2005) further showed that irrelevant items in WM influenced the fastest RTs and the first sac- cades in search, and the effect occurred even when the WM cue was always irrelevant. This last result suggests that an irrelevant WM stimulus can capture attention automatically. Other studies have demonstrated effects, even with pop-out targets (Soto, Humphreys, \& Heinke, 2006).

Interestingly, in many of these studies, the WM item constituted more of a "global" stimulus than the search target. For example, in Soto et al. (2005), the memory item was an outline shape and the search target was an oriented line that appeared within the outline shapes presented in the search display. It could be that the WM stimulus captures attention at least partly because there is a bias toward the global properties of a display, as suggested by the "global precedence" hypothesis (Navon, 1977), which holds that attention is biased toward global aspects of stimuli. When the WM cue matches early-emerging global properties of the search display, attention may be drawn to the global item matching the item in WM. It may also be that representing a stimulus in WM itself biases selection to the global level of a display, perhaps because the WM load reduces the resources available to process more local elements of a display. The biasing effects of WM on the selection of local and global properties of search displays were examined for the first time here. We had participants carry out a global-local task in which they had to detect a

J.-I. Kim, j.kim.1@bham.ac.uk; G.W.Humphreys, g.w.humphreys@bham.ac.uk 
target at either the local or the global level of a hierarchical compound shape (under divided attention conditions: Experiment 1), or they had to make a choice decision to a stimulus at one level (under focused attention conditions: Experiment 2). Prior to performing this task, participants had to hold an item in memory (as in Downing, 2000; Soto et al., 2005; Soto et al., 2006). We investigated whether this WM cue could bias the selection of hierarchical forms, and whether this bias was stronger to the global rather than the local level of the stimulus.

By investigating the selection of hierarchical forms, in this study we also examine whether irrelevant items in WM influence aspects of nonspatial attention. In prior studies, the WM stimulus has affected shifts of attention to a spatial location where the target could or could not fall. With global-local stimuli, though, selection may require more than fixing an "attentional window" on an area of space. Specifically, if an attentional window is fixed on a global shape, the local stimulus will be processed along with the global shape; selection of the global shape would require some other process, such as filtering by spatial features (see Hübner, 1993, 1996; Julesz \& Papathomas, 1984; Shulman, Sullivan, Gish, \& Sakoda, 1986). We examined whether stimuli in WM bias such nonspatial selection processes.

The factors affecting the processing of global and local shapes have been well documented over the past 30 years. Although numerous studies have demonstrated global precedence, with responses speeded to targets at global rather than local levels of form (see Kimchi, 1992, for a comprehensive review), other studies have shown that this depends on a range of factors, such as the density and spacing of the local elements (Huberle \& Karnath, 2006; Hughes, Fendrich, \& Reuter-Lorenz, 1990; Kimchi \& Palmer, 1982; Lamb \& Robertson, 1988), the overall size of the shape (Kinchla \& Wolfe, 1979), the familiarity of the local and global forms (Shalev, Humphreys, \& Mevorach, 2005), and so forth. Over and above this, it has generally been found that there is stronger evidence for global precedence when participants carry out target identification tasks in a distributed rather than in a focused mode of attention (Lux, Thimm, Marshall, \& Fink, 2006; Yovel, Yovel, \& Levy, 2001). This was assessed in the contrast between Experiments 1 and 2 here, with Experiment 1 using a distributed attention task ("Is a D present at either the local or global levels?") and Experiment 2 a focused attention task, in which the target had to be detected at a specific level ("Identify the local or the global form"). Does the contrast between distributed and focused modes of attention influence global precedence, and does this have an impact on the effects of attentional capture from stimuli in WM? Prior evidence suggests that attention capture from WM may be greater when the task demands a wide rather than a narrow focus of attention. Hernández, Costa, and Humphreys (2010) examined the effect of a WM cue on a search task in which participants first had to identify either the global shape of the array or the identity of a local item at fixation, prior to searching for a target. Effects of the validity of the WM cue (whether or not it matched the target) were greater when the global shape first had to be identified (when there was a wide span of attention) than when the local item had to be identified first (with a narrow focus of attention). Hernández et al. proposed that attentional guidance from WM is stronger when participants adopt a wide attentional window. Whether this is equivalent to adopting a distributed mode of attention was assessed here by comparing WM cuing under distributed and focused attention modes of a hierarchical form identification task. To evaluate the effects of WM on the selection of hierarchical stimuli, we had participants hold the identity of a letter in WM, then identify a target in a hierarchical (compound) letter. The WM cue could match a stimulus at one level of the hierarchical form, which could be the target (on valid trials) or a distractor (on invalid trials). There were also neutral trials in which the WM cue did not reappear in the hierarchical form. Effects of the WM cue would be shown if a validity effect emerged on performance. To test whether effects were due to the items in WM or to bottom-up priming from mere presentation of the cue, two experiments were run (Experiments $1 \mathrm{~B}$ and $2 \mathrm{~B}$ ) in which participants had to identify the cue but not hold it in memory. If bottom-up priming was critical, the data in the priming conditions (Experiments $1 \mathrm{~B}$ and $2 \mathrm{~B}$ ) should match those when the cue was held in WM (Experiments $1 \mathrm{~A}$ and $1 \mathrm{C}$; Experiment 2A). Interestingly, we report quite different patterns of data in the WM and priming conditions. In the WM conditions, having a cue that matched the distractor level of the form disrupted responses to the target. This effect was particularly strong under divided attention conditions. In contrast, in the priming condition, a cue that matched the distractor level facilitated responses to the target. These results highlight the contrast between processes that enhance perception by bottom-up priming and those that guide attention in a top-down manner through WM. In a third experiment, we tested whether the WM effects reflected the use of the hierarchical stimulus by participants to refresh their memory. As we elaborate in the General Discussion, the contrasting effects can be understood in terms of the neural structures underlying bottom-up and top-down search, which arise even without strategic refreshing of memory.

\section{EXPERIMENT 1A Working Memory, Hierarchical Stimuli, and Divided Attention}

\section{Method}

Participants. Twenty-five students and staff (age range, 19-35 years) at the School of Psychology of the University of Birmingham participated for course credit or cash. All had normal or correctedto-normal visual acuity.

Apparatus and Stimuli. The experiment was run on a Samsung SyncMaster 920N color monitor with a resolution of 1,280 $\times$ 1,024 pixels. The stimuli were produced using Adobe Flash (version CS3 Professional), and the test was programmed with E-Prime (version 2.0; Psychology Software Tools, Inc.). For each block, a set of different compound letters was constructed out of three English alphabet letters randomly selected from a group of letters (A, D, E $\mathrm{M}, \mathrm{N}, \mathrm{O}, \mathrm{S}, \mathrm{U}, \mathrm{V}$, and $\mathrm{X}$; for the examples of a set of compound letters used for a block of trials, see Figure 1). At a viewing distance of $70 \mathrm{~cm}$, the global letters subtended $8.5^{\circ} \times 7.3^{\circ}$ and the local letters $0.49^{\circ} \times 0.41^{\circ}$. The visual angle for the block letters (used for 


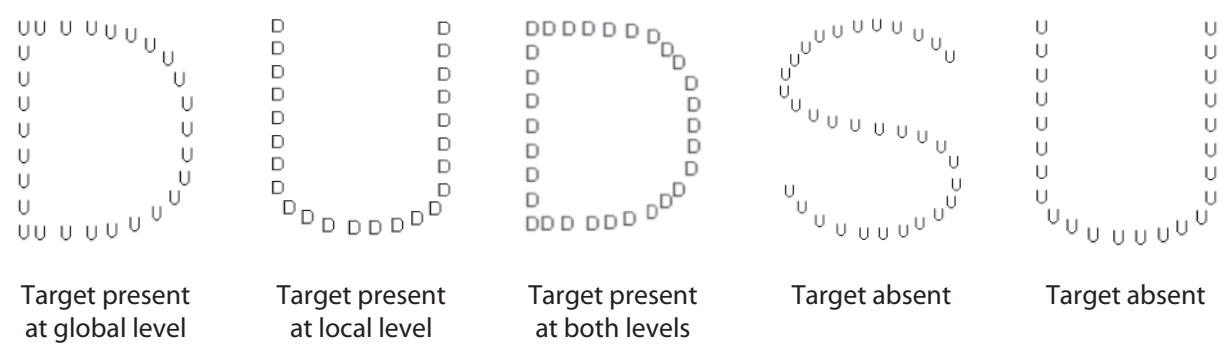

Figure 1. Examples of stimuli when target is D. All letters were in black displayed against a white background.

memory items) was $4.3^{\circ} \times 3.6^{\circ}$, which was of an intermediate size between the global and local letters of the compound stimuli. All stimuli were presented in the center of the screen. Response keys were located on the computer keyboard.

Design and Procedure. Figure 2 illustrates the experimental conditions and the sequence used in the present study. There were eight blocks of 62 trials. Each block contained trials from three conditions: valid (when the identity of the memory item matched that of the target item), invalid (when the identity of the memory item was different from the target and represented the stimulus at the other "distractor" level), and neutral (when the identity of the memory item was different from the target and was not present at the nontarget level). The target was always the letter D. Each trial, randomized within the block, began with a fixation cross displayed for $200 \mathrm{msec}$, followed for $500 \mathrm{msec}$ by a block letter that participants had to remember. There was a delay of $1,000 \mathrm{msec}$, a fixation cross for $200 \mathrm{msec}$, then the target was displayed for an unlimited period (until response). The task was to respond to the presentation of a target within a compound letter, irrespective of the level at which it was located. Memory probe questions on the to-be-remembered items occurred randomly, at the frequency of eight times per block, at the end of a trial. The memory probe asked participants to decide whether the letter shown was the same as, or different from, the one they held in their memory. Participants were warned about the memory test before the start of the experiment. The instructions em- phasized both the accuracy and the speed of response for both the target search and memory tasks. Participants had a practice session of 15 trials at the beginning of the experiment.

\section{Results}

In all the experiments, only RTs for correct responses in the global-local task were used in the analysis of the search responses. Trials with incorrect responses to memory probe questions, however, were included to secure the largest possible amount of data. The analyses were conducted separately for target-present data and target-absent data, since they had different conditions, with the targetabsent data having no valid trials.

Target-present data. The error rates for target detection and for memory probe responses were low at $4.1 \%$ and $6.2 \%$ on average, respectively (see Table 1 for the overall data summary).

Main effects. There was no evidence of a speed-accuracy trade-off, and the accuracy data were not analyzed further. A $2 \times 3$ ANOVA was carried out on the mean RT data for the global-local task. There were two factors: target level (global and local) and cue-target validity (valid, invalid,

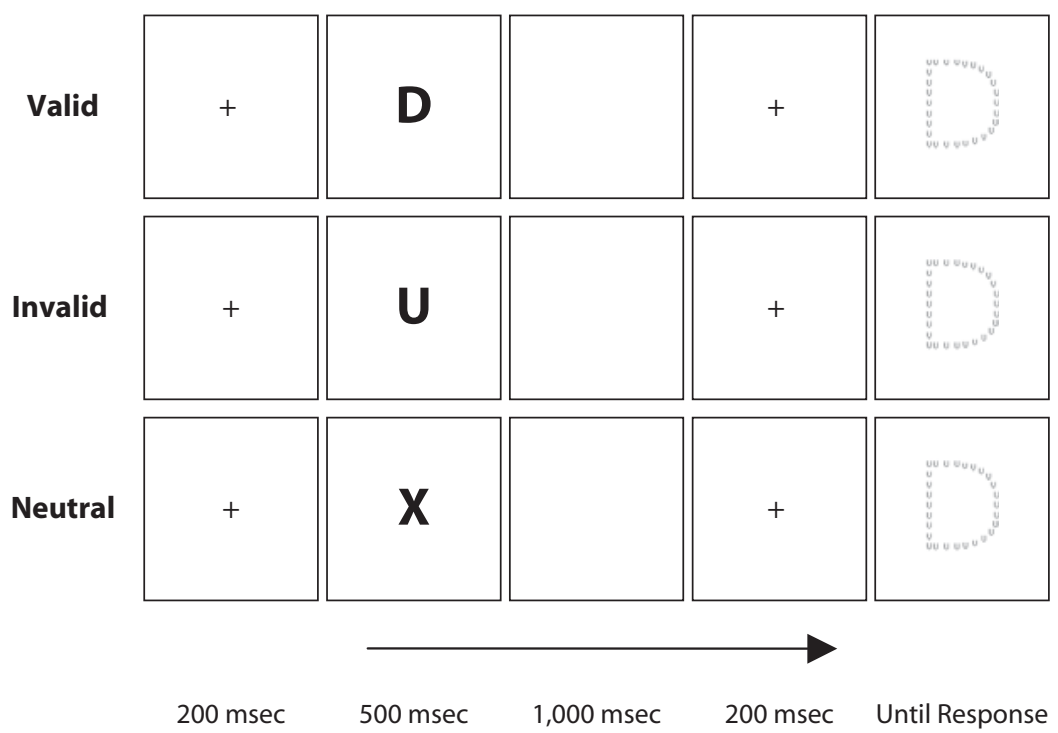

Figure 2. The sequence of events on a trial where the target was present at a global level. From top to bottom: Examples of valid, invalid, and neutral trials in a display. 
Table 1

Error Data on Target-Present Trials

in Experiments 1A (Memory) and 1B (No Memory)

\begin{tabular}{lllcc}
\hline Experiment & Level & Validity & $\begin{array}{c}\text { Search } \\
\text { Errors (\%) }\end{array}$ & $\begin{array}{c}\text { Memory } \\
\text { Errors (\%) }\end{array}$ \\
\hline \multirow{2}{*}{ Memory } & Global & Valid & 0.1 & 1.4 \\
& & Invalid & 0.0 & 1.2 \\
& Local & Neutral & 2.0 & 0.8 \\
& & Valid & 0.0 & 1.1 \\
& & Invalid & 0.0 & 1.1 \\
No memory & Neutral & 2.1 & 0.6 \\
& \multirow{3}{*}{ Global } & Valid & 0.5 & \\
& & Invalid & 1.0 & \\
& \multirow{3}{*}{ Local } & Neutral & 0.2 & \\
& & Valid & 0.1 & \\
& & Invalid & 0.7 & \\
& & Neutral & 0.4 & \\
\hline
\end{tabular}

and neutral). The results are shown in Figure 3. There was a strong effect of cue-target validity $[F(2,48)=17.90$, $p<.0001]$. Performance was better when the cue and the target were the same than when they were different. An effect of target level was not found, but there was a target level $\times$ cue-target validity interaction $[F(2,48)=$ $3.7, p=.037]$. Pairwise $t$ tests were conducted to contrast the conditions at the global and local levels separately. At the global level, there was a significant difference between the valid and invalid conditions $[t(24)=-4.95$, $p<.0001]$ and between the valid and neutral conditions $[t(24)=-4.56, p<.0001]$. The invalid and neutral trials did not significantly differ $[t(24)=1.56, p=.132]$. At the local level, there was a reliable difference between the valid and invalid conditions $[t(24)=-3.23, p=.004]$ and between the invalid and neutral conditions $[t(24)=$ $2.6, p=.015]$. The valid and neutral conditions did not differ $[t(24)=-1.34, p=.192]$. With valid cues, the responses to the target were significantly faster when it was shown at the global than at the local level $[t(24)=$ $-3.11, p=.005]$. The invalid and neutral trials did not differ across the local and global levels $[t(24)=0.84, p=$ .41 , and $t(24)=0.31, p=.76$, respectively]. To further assess the changes in the valid and invalid conditions for targets at global and local levels, cost-benefit analyses were performed, taking the differences between the valid and invalid conditions at the neutral baseline. The data were evaluated in a $2 \times 2$ ANOVA with the factors being levels of match to WM (global or local) and the cost-benefit (invalid-neutral or neutral-valid). There was a main effect of match $[F(1,24)=5.7, p=.025]$. No effect of validity was found $[F(1,24)=0.76, p=.39]$, and no match $\times$ validity interaction $[F(1,24)=2.1, p=.16]$. Costs and benefits were overall larger when the WM cue matched the global rather than the local letter.

Effect of congruency. The data were also analyzed to assess the effect of congruency between the global and local levels (when the two levels of each stimulus had the same identity vs. when they had different identities). Since there were no congruent trials in the invalid cue condition, invalid trials were not included.

Valid and neutral trials with congruent target stimuli were compared with those with incongruent target stimuli (see Figure 4 ) by conducting a $2 \times 3$ ANOVA with two cue-target validity factors (valid and neutral) and three global-local congruency factors (local letter incongruent with the target at the global level; global letter incongruent with the target at the local level; and congruent with the target at both levels). There were main effects of cuetarget validity $[F(1,24)=16, p=.001]$ and global-local congruency $[F(2,48)=4.8, p=.013]$. A cue-target validity $\times$ global-local congruency interaction was also found $[F(2,48)=4.1, p=.023]$.

For the valid and neutral conditions, $t$ tests were conducted. For the valid condition, there was a significant difference between the global match and local match trials $[t(24)=-3.1, p=.005]$ and between local match and congruent trials $[t(24)=2.35, p=.027]$. The difference between global match and congruent trials did not reach significance $[t(24)=1.6, p=.13]$. For the neutral condi-

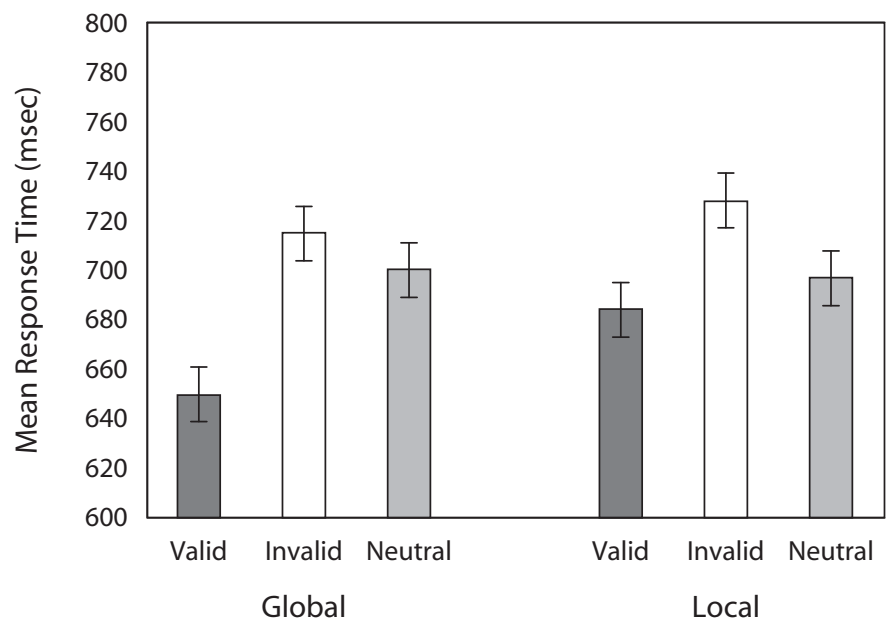

Figure 3. Experiment 1A: Effect of memory on target perception at different levels. Error bars represent standard errors of the means. 


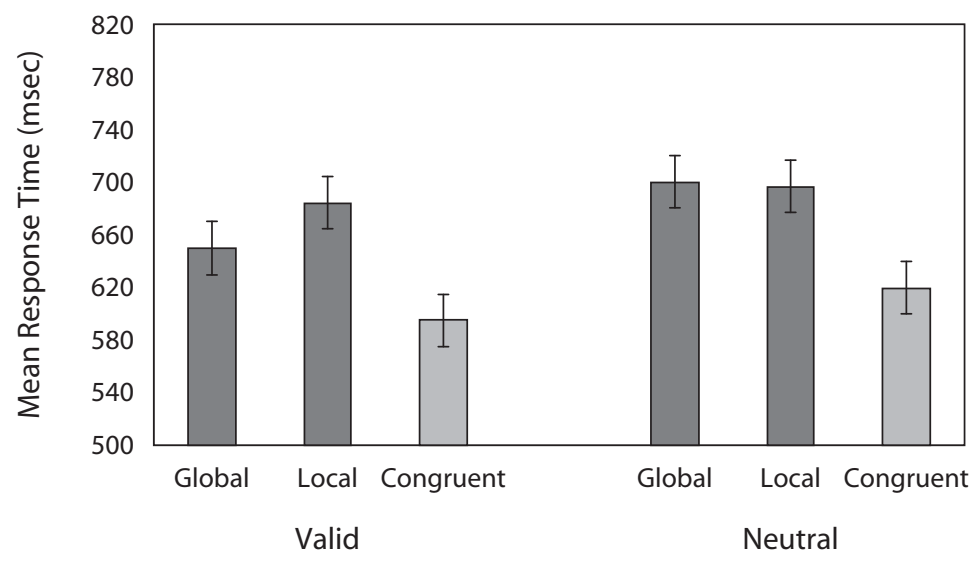

Figure 4. Effect of congruency of levels by validity. "Global" and "local" refer to the data from incongruent trials with the target at global and local levels, respectively. Error bars represent standard errors of the means.

tion, the difference was significant between global match and congruent trials $[t(24)=2.4, p=.026]$ and between local match and congruent trials $[t(24)=2.3, p=.032]$. There was no significant difference between global match and local match trials $[t(24)=0.31, p=.76]$. Congruent valid trials were also faster than congruent neutral trials $[t(24)=-2.1, p=.04]$; that is, there was an effect of the WM cue even when the letters making up the hierarchical form were congruent.

Target-absent data. The error rates were $3.2 \%$ for the search task and $4.2 \%$ for the memory task (see Table 2 for overall error data).

Main effects. There was again no evidence of a speedaccuracy trade-off (see Figure 5). A one-way ANOVA was conducted on the RTs, with the data sorted into three cue conditions: global cue, when the cue matched the global level of the compound letter; local cue, when the cue matched the local level of the letter; and neutral, when the cue did not match either level of the compound letter. There was a strong overall effect of validity $[F(2,48)=$ $10.1, p<.0001]$.

The difference between global cue trials and local cue trials was significant, according to $t$ tests $[t(24)=3.4, p=$ $.002]$. When compared with the neutral condition, the global cue condition was significantly slower $[t(24)=-4.5, p<$ $.0001]$, whereas the local cue and neutral conditions did not differ $[t(24)=-0.9, p=.4]$. Having the cue match a distractor at a global level slowed target-absent trials.

\section{Discussion}

Previous work has suggested that presenting items held in WM in a search display affects subsequent target selection (Downing, 2000; Pashler \& Shiu, 1999; Soto et al., 2005). In at least some of these studies, the WM item was a shape surrounding the target or distractor items in the search display. This means that the WM item was more global than the search stimuli, and this global status may be important for the effect. In other words, perhaps WM directs attention more easily to the global than to the local aspects of a display. The present study specifically exam- ined the effect of a WM stimulus on directing attention to the local or global level in a subsequent stimulus. When there was a global target, there was a strong effect of valid cuing from WM: RTs were faster to a global target, when it matched the identity of the cue, than when the cue was neutral and did not reappear in the following display. For local targets, valid trials were not reliably faster than neutral. In contrast, the costs of invalid cuing (when the cue matched the item at the distractor level) were reliable for local targets (match to global distractor) but not for global targets (match to local distractor). Overall, both the benefits from valid cuing to the target and the costs from invalid cuing to a distractor were greater when the cue matched a target/distractor at the global level. Interestingly, this result arose even though there was no overall global bias in the neutral condition; the bias to match the cue at a global level did not occur because that level was more salient than the local level. It reflected a more specific bias from WM to global level of stimulus representation. In the valid cue condition, the benefit from the cue could arise for two reasons: It could be because the cue guides attention to a matching stimulus or because the cue preactivates a response to the target. Note that on valid trials the cue had the identity of the target. The cost to target detection on invalid trials, though, cannot be due to response activation, since the cue then did not have the target's identity, nor did it activate an alternative response to the target.

Iable 2

Error Data on Target-Absent Trials in Experiments 1A and 1B

\begin{tabular}{lllcc}
\hline Experiment & Validity & Level & $\begin{array}{c}\text { Search } \\
\text { Errors (\%) }\end{array}$ & $\begin{array}{c}\text { Memory } \\
\text { Errors (\%) }\end{array}$ \\
\hline Memory & Valid & Global & 1.0 & 1.4 \\
& Leutral & & 1.1 & 1.7 \\
& Local & 1.1 & 1.1 \\
\multirow{2}{*}{ No memory } & Valid & Global & 1.3 & \\
& & Local & 1.4 & \\
& Neutral & & 1.2 & \\
\hline
\end{tabular}




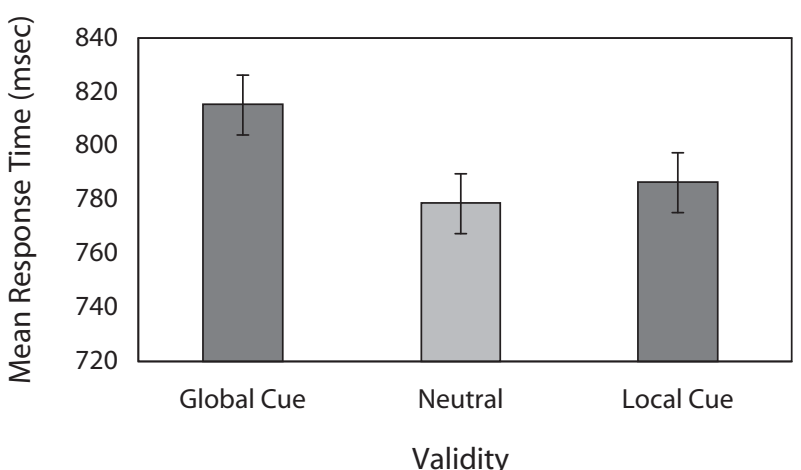

Figure 5. Experiment 1A: Effect of memory at different levels in target-absent trials. Error bars represent standard errors of the means.

We can conceptualize performance on invalid trials in two ways. One is in terms of the cost of attending to the incorrect level of the hierarchical form. A match between the WM cue and one of the letters in the hierarchical form could cue attention to the level where the match takes place. When the match is to a distractor, this would slow the selection of the target occurring at the other level of the form (on target-present trials). This attentional cuing account is similar to the argument put forward to explain the effects of a WM cue on directing spatial attention, although in that case it is argued that attention is misdirected to the wrong spatial location rather than to the wrong level of form (Soto, Hodsall, Rotshtein, \& Humphreys, 2008). An alternative proposal is that participants suppress the cue being held in WM (see Woodman \& Luck, 2007). Due to the suppression of the cue, RTs are slowed when the cue reappears in the hierarchical form. One difficulty for this account, however, is that there should be suppression of the WM cue on valid trials too, yet RTs to the hierarchical form were facilitated on such trials. It is also difficult to see why participants should suppress the item in WM, given that this item had to be maintained for the memory test. Another reason to query this account is that it does not fit with the neural data on the effects of repeating a WM cue in search (Soto, Humphreys, \& Rotshtein, 2007), which we discuss in more detail after Experiment 1B.

The data also indicate cost effects from re-presenting the cue on target-absent as well as on invalid targetpresent trials. On target-absent trials, an invalid cue may be disruptive because it draws attention to a matching item at one level of the stimulus, preventing participants from extracting partial cues for target absence from both levels. Another possibility is that, when the cue matches a stimulus at one level of the search display, a target-present response may be primed, which then has to be rejected for one to respond absent. However, as on target-present trials, the costs on absent responses were greater when the cue matched the global stimulus. In terms of response priming, it is not clear why matching the cue at the global level of the search display should disrupt performance. On the other hand, the greater global effect is consistent with the bias from WM to attend to the global level.
Not only was a cue-target validity effect found when the two levels of the hierarchical stimuli were incongruent, there were also effects of validity when the levels were congruent (valid RTs $<$ neutral RTs, for congruent stimuli). This validity effect even with congruent stimuli is notable, given that RTs to the target should be optimal in this case. Interestingly, the valid cuing of attention from WM to the global level of the target (with a local incongruent letter) led to RTs matching those when there was a valid global cue and the local and global levels were congruent. There was thus no reliable extra gain from having the local and global levels match the cue, relative to when only the global level matched.

Although in Experiment 1 participants were asked to hold the initial stimulus in WM, the cue was presented visually on each trial. This presentation could prime the participants' visual system, and this bottom-up priming could direct attention to a matching stimulus in the subsequent display. To test this bottom-up account, we conducted Experiment 1B, in which the memory requirement was taken out and the test was designed in such a way that participants were exposed to cues that had to be identified before they had to look for the target in the compound letter. Effects of bottom-up priming here should be matched to effects in Experiment 1A, when the cue had to be held in memory.

\section{EXPERIMENT 1B \\ Priming, Hierarchical Stimuli, and Divided Attention}

\section{Method}

Participants. Twenty-five students and staff (age range, 19-27 years) at the School of Psychology of the University of Birmingham participated for course credit or cash. All had normal or correctedto-normal vision.

Apparatus and Stimuli. The same apparatus as for Experiment $1 \mathrm{~A}$ was used. All the stimuli (block letters and compound letters) had the same properties as the ones specified in Experiment 1A.

Task and Procedure. There were eight blocks of 62 trials. Each trial started with an English alphabetical letter (the same block letters used for Experiment 1A) randomly chosen among four different ones used for a block. At the beginning of each block, one of the four letters was designated as a letter for a "no-go" trial, which signaled the participants to ignore the target in the following compound letter in the current trial. When the block letter was different from the designated one, participants were instructed to look for the target. The number of no-go trials was 18 per block. Again, the importance of both the accuracy and speed of response was emphasized.

\section{Results}

All the data were, again, divided into target-present and target-absent trials, as in Experiment 1A.

Target-present data. The error rate for the global-local task was $2.9 \%$ (see Table 1 for overall data summary).

Main effects. There was no sign of a speed-accuracy trade-off, and the accuracy data were not analyzed further. A $2 \times 3$ ANOVA was carried out on the mean RT data, with two target levels of stimulus (local and global) and three levels of cue-target validity (valid, invalid, and neutral) as factors. There was no effect of target level $[F(1,24)=3.17$, $p=.087]$, but there was a reliable cue-target validity effect 


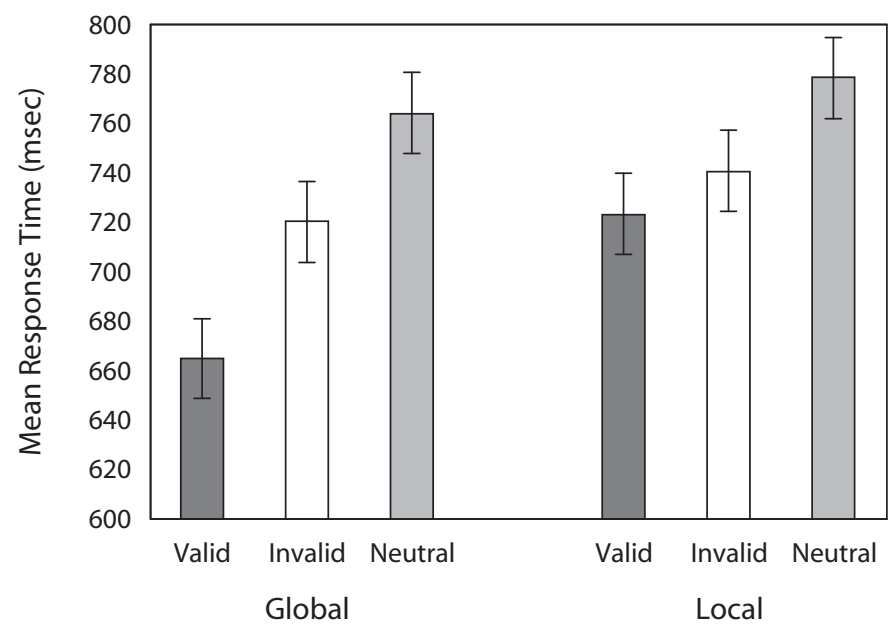

Figure 6. Experiment 1B: Effect of bottom-up priming on target perception at different levels of form. Error bars represent standard errors of the means.

$[F(2,48)=11.96, p<.0001]$. The interaction between target level and cue-target validity was not reliable $[F(2,48)=$ $2.44, p=.092]$. For valid trials, the data went in the same direction as in Experiment 1A. In particular, there was a stronger positive effect of validity for global targets, indicated by a cue-target validity $\times$ target level interaction, using data from just the valid and neutral trials $[F(1,24)=5.9, p=$ .023]. However, in contrast to Experiment 1A, invalid trials were faster than neutral trials $[F(1,24)=4.7, p=.039]$; this did not differ for local and global targets $[F(1,24)=0.057$, $p=.81]$. Mean RTs are shown in Figure 6.

Effect of congruency. The effect of congruency between the global and local levels was assessed by comparing trials with congruent and incongruent stimuli (all targetpresent). As in Experiment 1A, congruent trials were those with the target letter at both levels, and incongruent trials were those with the target letter at just the global or the local level. Performance was compared following valid and neutral cues (see Figure 7).
There were reliable effects of cue-target validity $[F(1,24)=42, p<.0001]$ and target level $[F(2,48)=7.8$, $p<.001]$. The RTs on valid trials were faster than on neutral trials. Averaging across the validity factor, congruent trials were faster than both local and global trials $[t(24)=$ $3.3, p=.003$, and $t(24)=2.4, p=.027$, respectively], and local and global trials did not differ $[t(24)=-2.0$, $p=.061]$. The cue-target validity $\times$ target level interaction was not reliable $[F(2,48)=2.2, p=.119]$.

Target-absent data. The error data are detailed in Table 2. There were no signs of a speed-accuracy tradeoff. The results from the analyses on target-absent trials contrasted with the effects found on target-present trials (see Figure 8). As in Experiment 1A, a one-way ANOVA was conducted with three levels of validity: global cue, local cue, and neutral. There was no effect of validity $[F(2,48)=0.17, p=.84]$.

Cross-experiment comparisons. To assess whether having to hold a stimulus in WM influenced selection,

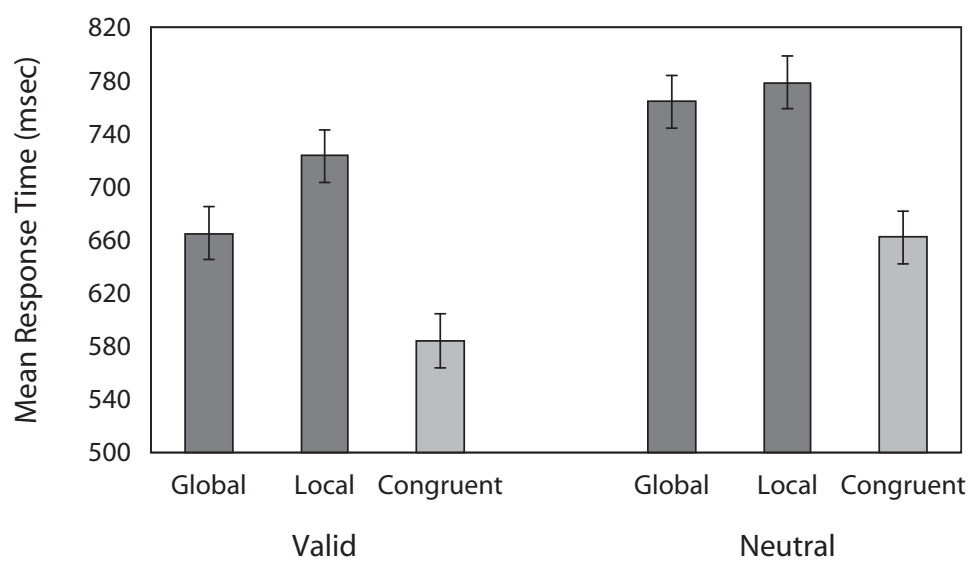

Figure 7. Experiment 1B: Effect of congruency for valid and neutral cue trials. "Global" and "local" here refer to the level at which the target appeared. Error bars represent standard errors of the means. 


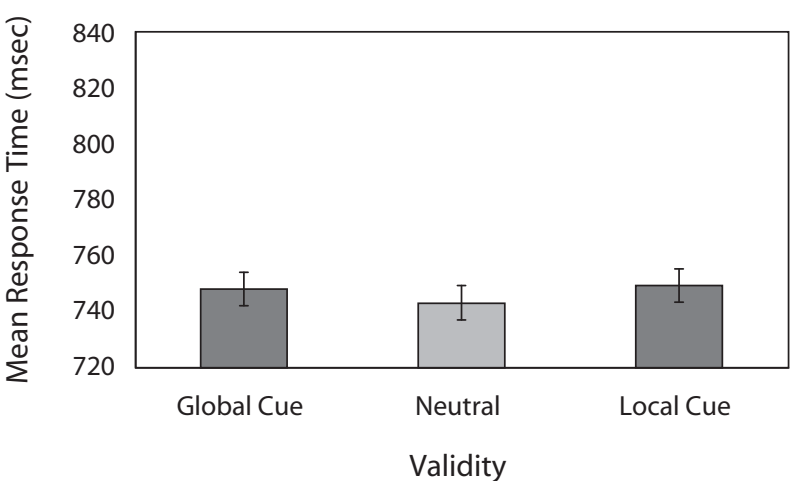

Figure 8. Experiment 1B: Effect of bottom-up priming on target-absent trials. Error bars represent standard errors of the means.

Experiments $1 \mathrm{~A}$ and $1 \mathrm{~B}$ were analyzed together. For the data from target-present trials, a mixed-design ANOVA was carried out, with one between-subjects factor (experiment) and two within-subjects factors (target level and cue-target validity). There was a reliable cue-target validity effect $[F(2,48)=15.6, p<.0001]$, but no overall effect of target level $[F(2,48)=3.55, p=.065]$. There were two-way interactions between cue-target validity and experiment $[F(2,48)=3.3, p=.04]$ and between target level and cue-target validity $[F(2,48)=10.8, p<$ $.0001]$, which were qualified by a target level $\times$ cuetarget validity $\times$ experiment interaction $[F(2,48)=3.4$, $p=.038]$. Performance was analyzed by separating the data for valid trials from the neutral baseline, and invalid trials from the neutral baseline across the experiments. First, a mixed-design ANOVA was conducted, with one between-subjects factor (experiment) and two withinsubjects factors (levels and validity: valid vs. neutral). The same ANOVA was then repeated for invalid versus neutral trials. For the contrast between valid and neutral trials, the results showed that there were significant main effects of cue-target validity $[F(1,48)=34.9, p<.0001]$ and target level $[F(2,48)=6.2, p=.016]$, but no effect of experiment $[F(1,48)=1.64, p=.206]$. However, there were interactions between cue-target validity and experiment $[F(1,48)=6.2, p=.017]$ and between target level and cue-target validity $[F(1,48)=14.6, p<.0001]$. There was no target level $\times$ cue-target validity $\times$ experiment interaction $[F(1,48)=0.08, p=.78]$. The effect of cue-target validity was larger in Experiment 1A (WM) than in Experiment 1B (priming) (77 vs. $32 \mathrm{msec}$ ). The cue-target validity effect overall was larger for global than for local targets (50 vs. $13 \mathrm{msec}$ ). For the contrast between invalid and neutral trials, there were no main effects (all $F$ s $<1$ ), but there was one substantial interaction between target level and experiment $[F(1,48)=10.1$, $p=.003]$. The cost for invalid versus neutral trials in Experiment 1A (52 msec, averaged across levels) became a benefit for invalid trials in Experiment 1B (40 msec, across levels; see contrasts reported in the individual experiments). The other interactions did not reach significance $($ all $F$ s $<1$ ).
A between-experiment analysis for target-absent trials was conducted with one between-subjects factor (experiment) and one within-subjects factor (validity: neutral, global valid, and local valid). There was no main effect of experiment $[F(1,48)=0.97, p=.33]$, but there was a reliable effect of validity $[F(2,48)=4.4, p=.014]$ and a validity $\times$ experiment interaction $[F(2,48)=3.1, p=$ .049]. The effect of validity was present in Experiment $1 \mathrm{~A}$ but not in Experiment 1B.

\section{Discussion}

In Experiment 1A, there were substantial effects of cue validity on responses to a hierarchical target letter, which were largest to items cued at the global level. Invalid cues also impaired performance. The effects of cuing arose on target-absent as well as on target-present trials. In Experiment $1 \mathrm{~A}$, participants had to memorize the cue. In Experiment 1B, participants had to identify the cue, but memorization was not required. Although there were effects of validly cuing attention to a target (relative to the neutral condition), any validity effects were smaller than in Experiment 1A. Perhaps even more strikingly, there was a qualitative shift in performance in the priming and memory conditions on invalid trials. Whereas previously an invalid cue disrupted target detection, in this priming experiment RTs to the target were facilitated when the cue matched the distractor at the nontarget level. In studies using brain-imaging methods, neural activations are found to be reduced when stimuli are repeated (e.g., Vuilleumier, Schwartz, Duhoux, Dolan, \& Driver, 2005). This repetition suppression effect may occur because stimuli are processed more efficiently when re-presented (see also Henson, Shallice, \& Dolan, 2000; Wig, Buckner, \& Schacter, 2009; Wiggs \& Martin, 1998). In the present study, the repeat of the cue at the distractor level of a hierarchical letter may enable the target letter to be processed more efficiently, speeding RTs. This effect of mere repetition of the distractor contrasts with the effects when the distractor was a repeat of a cue being actively held in memory, as in Experiment 1A. We have proposed that the cue in WM acts as a strong attractor of attention when a matching item appears in the hierarchical form, disrupting performance when the cue is invalid. Alternatively, there is suppression of the cue in WM, which slows performance when the cue reappears in the hierarchical form. In either case, these effects seem confined to situations in which the cue is held in WM.

These behavioral data are particularly striking in relation to work on the neural substrates of attention capture from WM. Soto et al. (2007) used fMRI to measure neural activation during a search task when participants either merely identified a cue or held it actively in WM. When the cue was held in memory, then reappeared in the search display, there was enhanced activation in brain areas sensitive to stimulus repetition (e.g., the parahippocampal gyrus and the superior frontal gyrus). This occurred on invalid as well as valid trials. This top-down increase in activation may serve as the neural basis for attentional capture. For example, increased activation when the cue reappears could act to drive attention to the cue. This 
Table 3

Error Data on Target-Present Trials in Experiment 1C

\begin{tabular}{ccccc}
\hline Experiment & Level & Validity & $\begin{array}{c}\text { Search } \\
\text { Errors (\%) }\end{array}$ & $\begin{array}{c}\text { Memory } \\
\text { Errors (\%) }\end{array}$ \\
\hline \multirow{2}{*}{ Memory } & Global & Invalid & 0.3 & 1.0 \\
& & Neutral & 0.2 & 0.4 \\
& Local & Control & 1.3 & 0.8 \\
& & Invalid & 1.5 & 0.9 \\
& Neutral & 0.3 & 1.3 \\
& Control & 1.5 & 0.6 \\
\hline
\end{tabular}

would be consistent with an attentional capture account of the data on invalid trials in Experiment 1A. In contrast, when the cue was identified but not held in memory, these same neural areas showed evidence of repetition suppression (reduced activation when the cue reappeared in the search display compared with when it did not reappear). This repetition suppression effect may provide the neural basis of facilitated perception of a repeated cue, found on invalid priming trials here. Bottom-up activation of cued representations may facilitate perceptual encoding without exerting a strong effect on attention.

\section{EXPERIMENT 1C Working Memory, Hierarchical Stimuli, and Automatic Guidance}

In Experiments $1 \mathrm{~A}$ and $1 \mathrm{~B}$, the initial cue was valid on $33 \%$ of the trials. It is possible that this could have encouraged participants to match the cue to the target, even when the cue was valid and invalid equally often. If the tendency to match the cue deliberately was increased when the cue was also held in WM, this would account for the larger cuing effects in the WM condition (Experiment 1A). In experiments using spatial search rather than hierarchical letter perception, Soto et al. (2005) showed that cuing effects from WM occurred even when the cues were never valid (the cue was always invalid when it reappeared in the search display). Soto and Humphreys (2007) reported similar results when a verbal WM cue was presented. They argued that the cue automatically directed visual at- tention even when it was always invalid. Experiment $1 \mathrm{C}$ tested for a similar automatic effect from WM on attention to the level of hierarchical forms. To do this, Experiment $1 \mathrm{~A}$ was repeated, but with only invalid and neutral trials included.

\section{Method}

Participants. Twenty-five students (age range, 19-28 years) at the School of Psychology of the University of Birmingham participated for course credit. All had normal or corrected-to-normal vision.

Apparatus and Stimuli. The same apparatus from Experiments $1 \mathrm{~A}$ and $1 \mathrm{~B}$ was used. All stimuli (block letters and compound letters) had the same properties as the ones specified in the previous experiments.

lask and Procedure. The task sequence was the same as the one in Experiment 1A. The letter D remained the target, and two other alphabetical letters, $\mathrm{U}$ and $\mathrm{X}$, were used to construct compound letters. For the memory cue, three letters, U, X, and N, were used. The important change was that valid trials, in which the identity of the memory item matched that of the target, were not included in this experiment, and only invalid and neutral conditions were maintained. Additionally, there was a new type of trial in which the identity of the memory item was never present at either level of the compound letter (e.g., when $\mathrm{N}$ was the memory item). This new control condition tested whether there was any effect of having the memory cue as a letter that could sometimes appear in the hierarchical letter display, even when it was not actually present on the trial (on neutral trials). There were, in total, three experimental conditions in the present test (invalid, neutral, and control); four blocks of 72 trials were run.

\section{Results}

Target-present data. The error rates were 5.1\% and $5 \%$ for the search and memory tasks, respectively (see Table 3).

Main effects. There was no sign of a speed-accuracy trade-off and no sign of any effect of validity on memory trials. These data were not analyzed further. Figure 9 gives the overall RT data. A 2 (target level) $\times 3$ (cue-target validity) ANOVA showed a reliable effect of target level $[F(1,24)=$ $22.1, p<.0001]$ but no cue-target validity effect $[F(2,48)=$ $0.385, p=.698]$. There was no target level $\times$ cue-target validity interaction $[F(2,48)=0.02, p=.98]$.

Effect of congruency. The data were analyzed to assess the effect of congruency between the local and global lev-

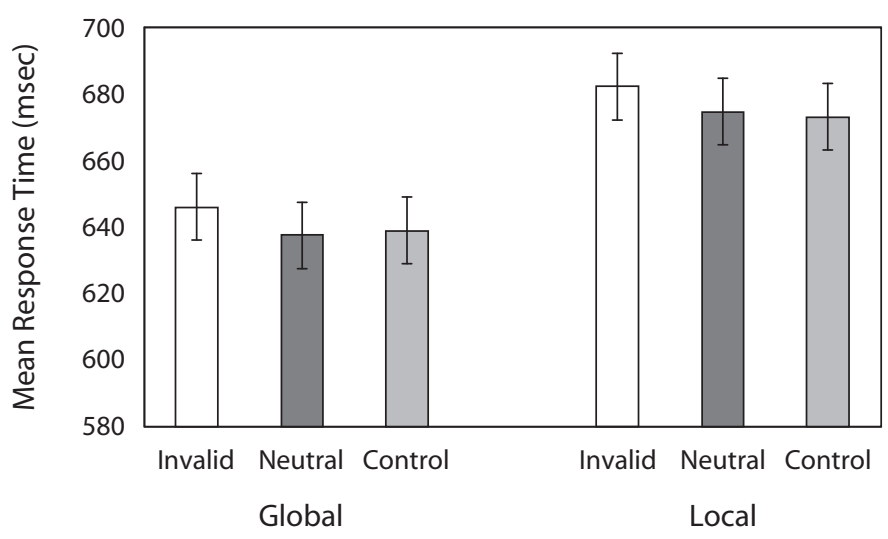

Figure 9. Effect of working memory on target perception at different levels of form in Experiment 1C (no valid trials). Error bars represent standard errors of the means. 


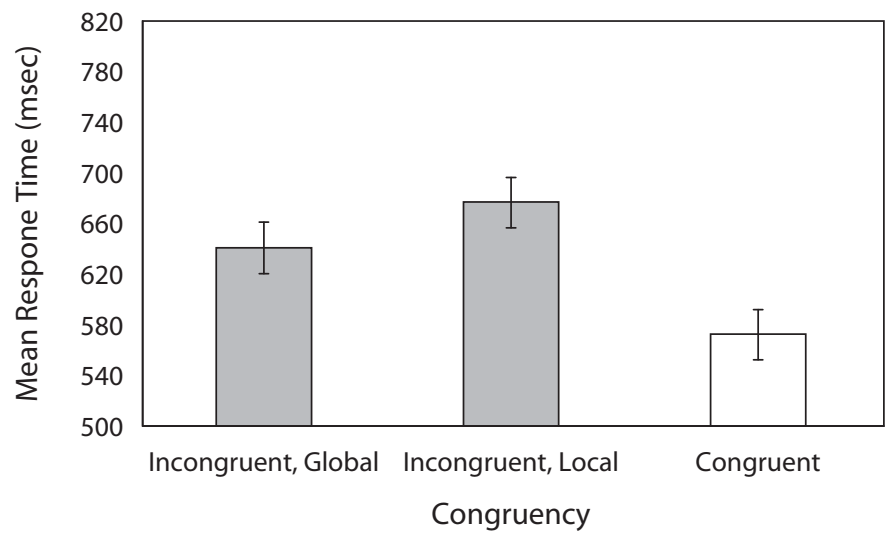

Figure 10. Experiment 1C: Effect of congruency. "Global" and "local" refer to the level at which the target was located. Error bars represent standard errors of the means.

els (see Figure 10). Since there were no valid trials here, the data were based on neutral cue trials only. Averaging across the cue-target validity factor, all incongruent trials were analyzed against congruent trials via a one-way ANOVA. There was a reliably significant effect of globallocal congruency $[F(2,48)=8.9, p<.0001]$. Pairwise $t$ tests showed significant differences between congruent trials and incongruent trials with the target at both the global and local levels $[t(24)=2.3, p=.03$, and $t(24)=$ $3.4, p=.002$, respectively]. RTs for incongruent trials with a target at a global level (WM match to the local level) were significantly faster than RTs for incongruent trials with a target at a local level (WM match to the global level) $[t(24)=-4.7, p<.0001]$.

Target-absent data. The error rates were $2.9 \%$ for the search task and 3.9\% for the memory task (see Table 4). Again, there was no effect of validity on memory. The data from target-absent trials were analyzed (see Figure 11), with the data separated according to whether the invalid cue matched the global or local level of the hierarchical letter by carrying out a one-way ANOVA on the three validity conditions. There was an effect of validity $[F(2,48)=5.9, p=.005]$. In each of the two invalid conditions, RTs were significantly larger than in the neutral condition $[t(24)=3.3, p=.003$, for global matches, and $t(24)=3, p=.006$, for local matches]. There was no difference between the global and local match conditions on invalid trials $[t(24)=-0.33, p=.74]$.

\section{Discussion}

Unlike in Experiment 1A, there was no effect of the validity of the cue on target-present responses. However, there was an effect on target-absent responses: RTs were slowed when the cue in WM matched the identity of the letter at one level of the hierarchical form. Interestingly, the magnitude of this effect on absent trials was similar to that in Experiment 1A (the difference between the neutral and global match conditions here was $39 \mathrm{msec}$, whereas this difference was $36 \mathrm{msec}$ in Experiment 1A). In addition, on trials where the cue matched one level of an incongruent distractor, RTs were faster when there was a local rather than a global match. These results are consistent with there being an automatic effect on directing attention to one level of a hierarchical form when it matched an item held in WM, affecting performance even when the WM cue was always invalid. The effect was again greater when the invalid cue matched the global level of form. An alternative is that the WM was suppressed and this slowed RTs, but there is no reason to expect such an effect to be most pronounced for cues reappearing at the global level.

The argument that there is an automatic guidance effect on attention from items held in WM is not without controversy. For example, Woodman and Luck (2007) examined effects of a stimulus in WM on spatial search. The item in memory never matched the search target. Unlike Soto et al. (2005), they found that RTs were actually speeded when the memory cue reappeared as a distractor in the search display. Woodman and Luck proposed that participants could bias themselves against items held in WM, so that targets were selected more easily when the WM matched a distractor rather than a target (speeding RTs on invalid relative to valid trials). Olivers (2009) has presented data suggesting that whether a bias is set against an irrelevant item in WM depends on several factors, such as whether the target changes across trials (the bias against the WM item is found with varied rather than consistent mapping; with consistent mapping of the search target, attention is biased to the WM cue). Han and Kim (2009) have also shown that a positive bias to an item re-presented from WM can switch to a negative bias against it when the WM item is always invalid and when there is a relatively long interval between the cue and the search display (1,000 msec or more). The present study used consistent

Iable 4

Error Data on Target-Absent Trials in Experiment 1C

\begin{tabular}{ccccc}
\hline Experiment & Validity & Level & $\begin{array}{c}\text { Search } \\
\text { Errors (\%) }\end{array}$ & $\begin{array}{c}\text { Memory } \\
\text { Errors (\%) }\end{array}$ \\
\hline \multirow{2}{*}{ Memory } & Invalid & Global & 0.8 & 1.4 \\
& & Local & 1.0 & 1.4 \\
& Control & & 1.1 & 1.1
\end{tabular}




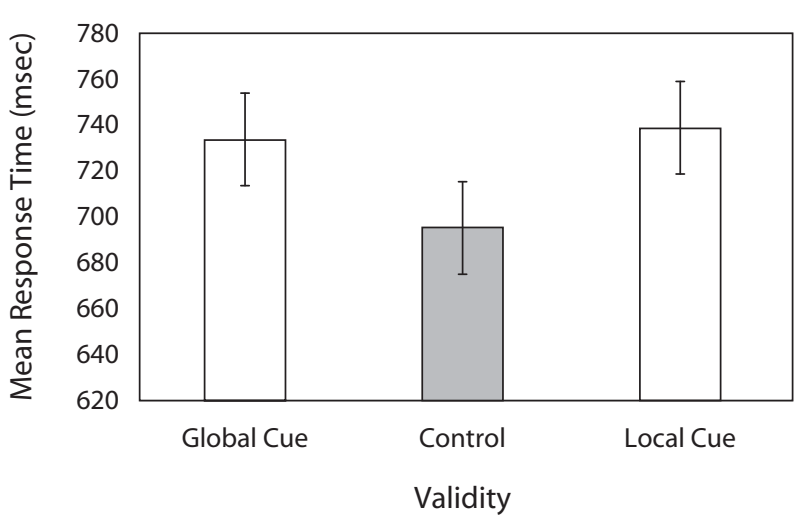

Figure 11. Experiment 1C (no valid trials): Effect of memory on target perception at different levels on target-absent trials. Error bars represent standard errors of the means.

mapping (favoring a bias to the WM stimulus) but had a relatively long interval between the onset of memory cue and the onset of the hierarchical letter $(1,500 \mathrm{msec})$. This long interval could have enabled participants to begin to set themselves against the (always invalid) WM cue, which would reduce the strength of the positive bias to the WM item, compared with when the item was sometimes valid.

One other argument that can be raised against the cue in WM automatically affecting attentional guidance is that participants might use the cue to deliberately refresh their memory by attending to the cue's reappearance in the hierarchical form. According to this "memory refresh" account, WM performance ought to be better when the cue reappears in the display (on valid and invalid trials) than when it does not (neutral and control trials). There was no evidence for this (Tables 1-4). Nevertheless, to test this proposal we conducted Experiment 3, which used conditions in which it would not benefit participants to attend to the hierarchical stimulus to refresh their memory. These conditions should make it difficult to argue that any effects reflect memory refreshing.

\section{EXPERIMENT 2A \\ Working Memory, Hierarchical Stimuli, and Selective Attention}

There were several interesting results in Experiment 1. First, in Experiment 1A, baseline responses with hierarchical letters did not show a global precedence effect, but evidence for the effect emerged when there was a match between a cue held in WM and the hierarchical form. There were stronger positive cuing effects (on valid trials) to global targets, and a greater cost to local targets from invalid cuing trials (when the cue matched the global form). This suggests that attentional guidance from WM is globally biased. In addition, there was evidence that effects from items held in WM and from bottom-up priming from the cue may be qualitatively different (Experiments $1 \mathrm{~A}$ and 1B). Bottom-up priming facilitated perceptual processing without biasing attention. In contrast, either items in WM bias attention to a matching form or these items are suppressed. Evidence from fMRI is more consistent with the attentional bias account (Soto et al., 2007), and this account also better explains why distractors are most disruptive when they appear at a global level, if the WM stimulus biases attention to that level.

Experiment 1 used a divided attention procedure, whereby participants had to look for the target letter at both levels. Divided attention may encourage attentional deployment at a global level. Experiment 2 used a selective attention format, whereby participants were given the specific level to be attended to. In this task, participants have the opportunity to filter out the letter at the task-irrelevant level. Previous studies have indicated that the selective attention procedure tends to "weaken" differential hierarchical perception by enabling participants to focus on one specific level while ignoring the other (Lux et al., 2006; Yovel et al., 2001). We ask whether matches to a WM item bias attention to the global level even when participants attempt to attend to just the local level. A positive effect of the cue here would indicate that its effect may counteract the focused attentional bias to the target level.

The design of Experiment 2 also meant that we could test for effects of response priming on performance. The effects of valid cuing in Experiment 1 could at least in part reflect priming of a target-present response, since the valid prime had the same identity as the target. Experiment 2 included conditions in which the search letter had the same (congruent) letter at the global and local levels, and where the nontarget level was non-response-related (control stimuli; see Figure 12). In the congruent WM condition, the prime was coded as valid (the cue had the same identity as the stimulus at the target level) or neutral (the cue was not a member of the response set), or it was the response alternative to the stimulus present at the target level (we term this the invalid-response condition). If there is an effect of response priming, the RTs should be slower here in the invalid-response condition than when the cue is neutral (response unrelated). Note that the prime is not present at either level of the target letter. In the control WM condition, the cue was coded as either valid (cued the target letter), invalid-response (cued the alternative response but was not present in the hierarchical target letter), or invalid (as in Experiment 1). In the invalid WM condition, the cue was not a response alternative but matched the distractor in the hierarchical target letter. Effects of response priming and invalid attention cuing can be contrasted by comparing these last two conditions. There were also incongruent stimuli, in which the distractor level of the hierarchical stimulus demanded the opposite response to the target. These stimuli could be preceded by valid primes (matching the target), neutral primes (not present in the hierarchical stimulus), or invalid-response and invalid primes (which both cued the alternative response to the target and also matched the stimulus at the distractor level of the hierarchical letter).

\footnotetext{
Method

Participants. Twenty-five students and staff (age range, 19-42 years) at the School of Psychology of the University of Birmingham
} 
Congruent

Incongruent

\section{Control}

\section{Congruent}

Incongruent

\section{Control}

D

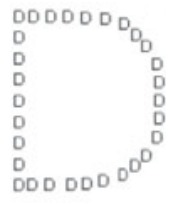

Valid

D

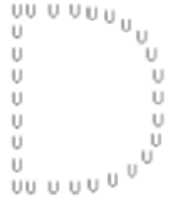

Valid

D

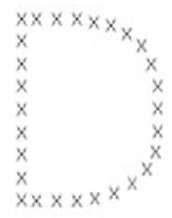

Valid

\section{Target at Global Level}

$\mathbf{U}$

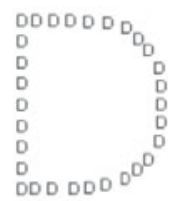

Invalid-Response

$\mathbf{U}$

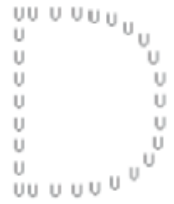

Invalid and Invalid-Response

$\mathbf{U}$

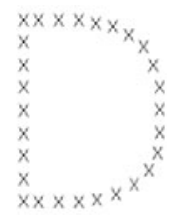

Invalid-Response

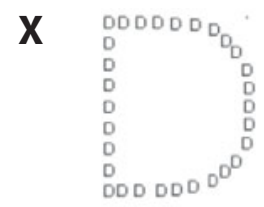

Neutral

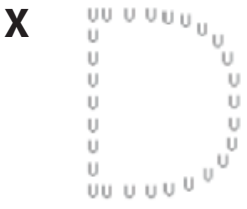

Neutral

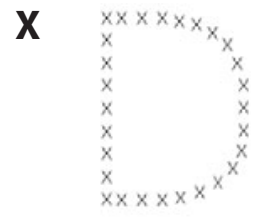

Invalid

\section{Target at Local Level}

D

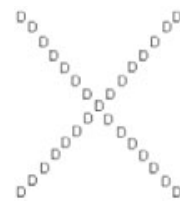

D

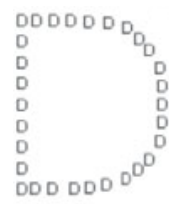

Valid

D

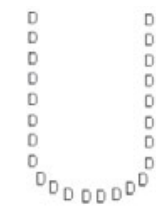

Valid

Valid
$\mathbf{U}$

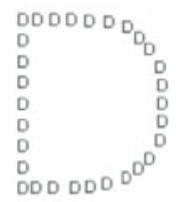

Invalid-Response

$\mathbf{U}$

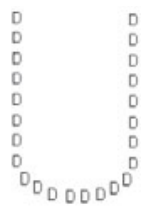

Invalid and Invalid-Response

$\mathbf{U}$

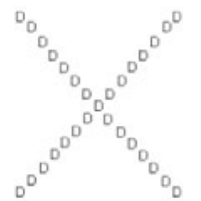

Invalid-Response
$\mathbf{X}$

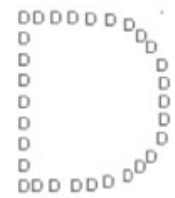

Neutral

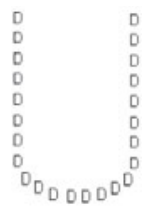

Neutral
$\mathbf{X}$

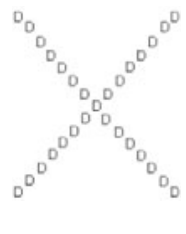

Invalid

Figure 12. Hierarchical targets employed in Experiment 2A, shown here to illustrate the type of target (congruent, incongruent, control) and the different working memory conditions (valid, invalid, neutral, invalid-response, and invalid and invalid-response). Targets were $D$ and $U$. The examples illustrated here are when the target is $D$. The small block letter illustrates the prime on that trial. 
participated for course credit or cash. All had normal or correctedto-normal visual acuity.

Apparatus and Stimuli. The test equipment and stimuli were the same as in Experiment 1. Three letters - D, U, and X-were used to construct the compound stimuli.

Task and Procedure. Fourteen blocks ( 7 global and 7 local tasks, randomized) of 18 trials were run with each participant. Figure 12 presents an illustration of the stimuli used in the present experiment. As a new block began, one specific level was cued by the word local or global to indicate the level to be attended to across the following trials. The trials had the same features as the ones from Experiment 1. Randomized within the block, each trial began with a fixation cross, which was followed by a block letter for the participants to remember. After a delay of $1,000 \mathrm{msec}$ and a fixation displayed for $200 \mathrm{msec}$, a compound letter was presented for response. Memory probe questions were presented randomly at the ends of trials. There were three congruency conditions, determined by the relations between the local and global levels of a single stimulus (see Figure 12).

The local and global levels of the hierarchical letters could be congruent (when the global and local levels were the same letter, either $\mathrm{D}$ or $\mathrm{U}$ ), incongruent (when the global and local levels were different letters, $\mathrm{D}$ and $\mathrm{U}$ ), and control (when one of the levels was a non-response-related one, the letter X). Within each congruency condition there were three cue-target validity conditions (valid, invalid, and neutral), defined by the relation between the cue and the subsequent hierarchical stimulus. Note that for the congruent condition, there was not a nontarget level, and so there could be no invalid cuing trials (although there could be invalid-response trials). The targets were always the letters D and U. Participants had to decide whether the letter present at the task-relevant level was D or U and to press the response keys (left or right arrow keys) on the keyboard, as instructed.

\section{Results}

The search error rate was $6.3 \%$, and the memory error rate was $6.3 \%$ (see Table 5 for the data summary). Four separate ANOVAs were conducted to test the overall congruency effect in the neutral conditions, and to test the validity effect under each congruency condition (congruent, incongruent, and control).

Congruency effect. The effect of global-local congruency was assessed by taking the data when there was a neu- tral memory cue. The data were analyzed in a 2 (target level: global and local) $\times 2$ (global-local congruency: congruent and incongruent) ANOVA. The results (see Figure 13) showed that there was a reliable global-local congruency effect $[F(1,24)=21, p<.0001]$. There was no effect of target level $[F(1,24)=0.23, p=.63]$ and no target level $\times$ global-local congruency interaction $[F(1,24)=0.25, p=$ $.62]$. The magnitude of the congruency effect was the same for targets at the local and global levels.

Validity effect under each level of congruency condition. Separate $2 \times 3$ ANOVAs were carried out for each global-local congruency condition (for congruent, incongruent, and control stimuli), to test the relations between target level (global and local) and cue-target validity (valid, neutral, and the various invalid conditions). Figure 14 shows the overall data.

Congruent displays. There were no effects of cue-target validity or target level, and no interaction (all $F \mathrm{~s}<1$ ).

Incongruent displays. There were again no reliable effects (all $F$ s $<1$ ).

Control displays. There was a main effect of cue-target validity $[F(2,48)=5.6, p=.007]$. There was no effect of target level and no interaction (both $F \mathbf{s}<1$ ).

Pairwise comparisons for control stimuli were conducted to assess the main effect of cue-target validity, with the data averaged across the target level (global and local). There were significant differences between the valid and invalid conditions $[t(24)=-2.1, p=.045]$ and between the invalid and invalid-response conditions $[t(24)=-3.0, p=.007]$. The valid and invalid-response conditions did not differ significantly $[t(24)=0.83, p=$ .41]. The absence of a difference between the valid and invalid-response conditions indicates that response priming (in the invalid-response condition) did not strongly modulate responses to the target. It also suggests that there was little benefit from valid priming, presumably because attention was already set to the target level. However, RTs were slowed on invalid trials. This suggests that the WM

Iable 5

Error Data in Experiment 2A

\begin{tabular}{|c|c|c|c|c|c|}
\hline Experiment & Level & Congruency & Validity & $\begin{array}{c}\text { Search } \\
\text { Errors }(\%)\end{array}$ & $\begin{array}{c}\text { Memory } \\
\text { Errors (\%) }\end{array}$ \\
\hline \multirow[t]{18}{*}{ Memory } & \multirow[t]{9}{*}{ Global } & \multirow[t]{3}{*}{ Congruent } & Valid & 0.0 & 0.0 \\
\hline & & & Invalid-response & 0.0 & 1.0 \\
\hline & & & Neutral & 0.0 & 0.0 \\
\hline & & \multirow[t]{3}{*}{ Incongruent } & Valid & 1.4 & 0.0 \\
\hline & & & Invalid & 0.8 & 0.6 \\
\hline & & & Neutral & 1.1 & 0.2 \\
\hline & & \multirow[t]{3}{*}{ Control } & Valid & 0.3 & 0.2 \\
\hline & & & Invalid-response & 0.0 & 0.3 \\
\hline & & & Neutral & 0.0 & 0.7 \\
\hline & \multirow[t]{9}{*}{ Local } & \multirow[t]{3}{*}{ Congruent } & Valid & 0.0 & 0.2 \\
\hline & & & Invalid-response & 0.0 & 1.0 \\
\hline & & & Neutral & 0.0 & 0.0 \\
\hline & & \multirow[t]{3}{*}{ Incongruent } & Valid & 1.1 & 0.3 \\
\hline & & & Invalid & 1.0 & 0.3 \\
\hline & & & Neutral & 0.4 & 0.2 \\
\hline & & \multirow[t]{3}{*}{ Control } & Valid & 0.1 & 0.9 \\
\hline & & & Invalid-response & 0.0 & 0.3 \\
\hline & & & Neutral & 0.1 & 0.2 \\
\hline
\end{tabular}




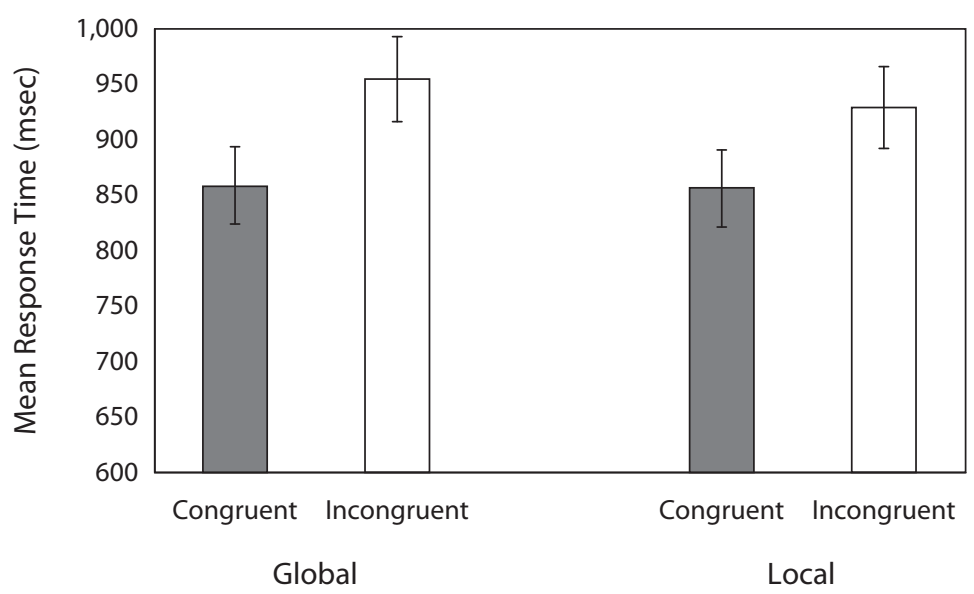

Figure 13. Experiment 2A: Overall congruency effect by level. Error bars represent standard errors of the means.

cue may still direct attention to the distractor level, even when participants are in a focused attention mode.

In order to assess how the effects of the memory cue varied across the divided and focused attention conditions, the data from the valid and invalid conditions in Experiment $1 \mathrm{~A}$ were compared with those from Experiment 2A, with experiment as a between-subjects factor. ${ }^{1}$ There was a main effect of cue-target validity $[F(1,48)=15.95, p<$ $.0001]$, which did not interact with experiment $(F<1)$.

\section{Discussion}

In Experiment 2, with control stimuli, we again found a cuing effect, with RTs being slowed on invalid trials compared with the other conditions (valid and invalidresponse, in this case). This cost effect (relative to invalidresponse trials) was similar to that found in Experiment 1A (and the overall validity effect did not differ across the experiments). The data suggest that the WM cue could still attract attention to a matching distractor at the nontarget level, even though participants could focus attention at one level here. The alternative account is that the WM cue was suppressed and this slowed the processing of the hierarchical stimulus. However, we would expect RTs to be slowed on valid trials, too, in this case, and there was no evidence for this.

The present results appear at first sight to counter those of Hernández et al. (2010), who reported stronger effects of a WM cue on subsequent visual selection when participants adopted a broad window of attention. However, it may be that adopting a broad window of attention (as in Hernández et al., 2010) is not the same as being in a distributed versus focused mode of attention (contrasting Experiments 1 and 2). Even when participants could focus attention in advance here, they would still have to adopt a broad window of attention for the global form. Rather than corresponding to a narrow attentional window, focused attention here may involve tuning visual filters to a particular spatial frequency, and this tuning may operate even when a broad window of attention is adopted for a global target. The present data indicate that, even if par- ticipants focus attention on a target spatial frequency, they can still be cued to attend to the other level of the stimulus, if that level matches an item in WM.

The present data also suggest that the beneficial effects of validity are reduced when participants operate in a focused mode of attention (note the lack of difference between the valid and invalid-response conditions for control stimuli here). This would fit with the validity benefit occurring because attention is attracted to the appropriate level of the stimulus when the WM is valid and attention is distributed (Experiment 1A). Validity effects are reduced when the appropriate level of the target is already selected (in the focused attention mode). The failure to find a cost on invalid-response trials, compared with valid trials (e.g., for the control stimuli), also suggests that response priming effects were not strong (given that the response priming could occur on valid trials). Effects of valid and invalid cues did not appear to operate at a response level.

We failed to find effects of the cues here with congruent and incongruent hierarchical forms, although we did find that RTs were generally slower with incongruent stimuli. With these stimuli, the time taken to select the response to the target could have "washed out" any effects of the cue.

Experiment 2B replicated Experiment 2A, but used the procedure employed in Experiment 1B, in which participants identified primes but did not have to hold them in memory. This experiment then assessed effects of bottom-up priming from the cue. In Experiment 1B, we found that RTs were speeded when the cue was present in the target letter, both when it matched the target (on valid trials) and when it matched the nontarget (on invalid trials). Would a similar effect emerge here in the invalid condition, even when participants could focus their attention on the target level?

\section{EXPERIMENT 2B}

\section{Working Memory, Hierarchical Stimuli, and Selective Attention}

\section{Method}

Participants. Twenty-five students and staff (age range, 19-27 years) at the School of Psychology of the University of Birmingham 

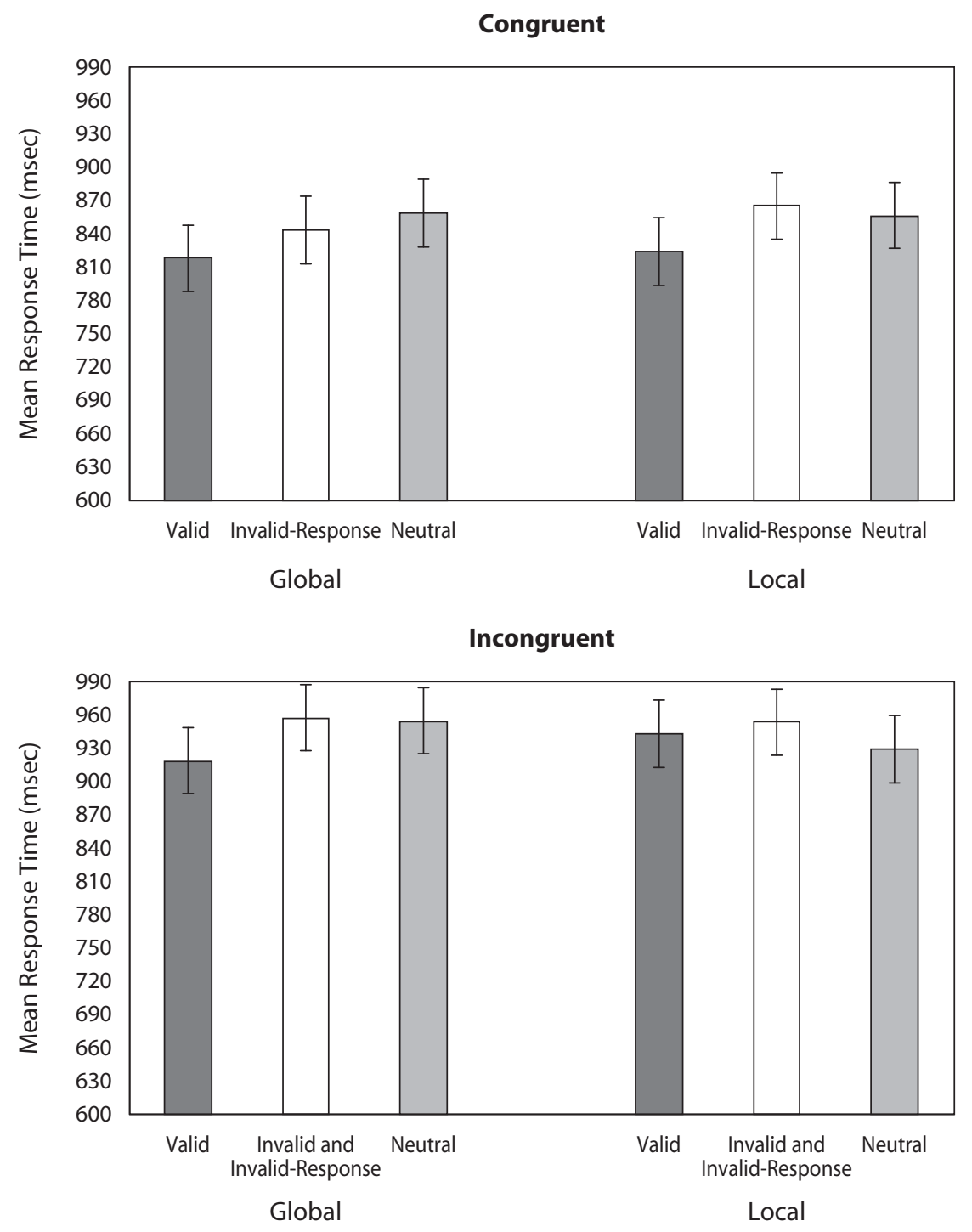

Control

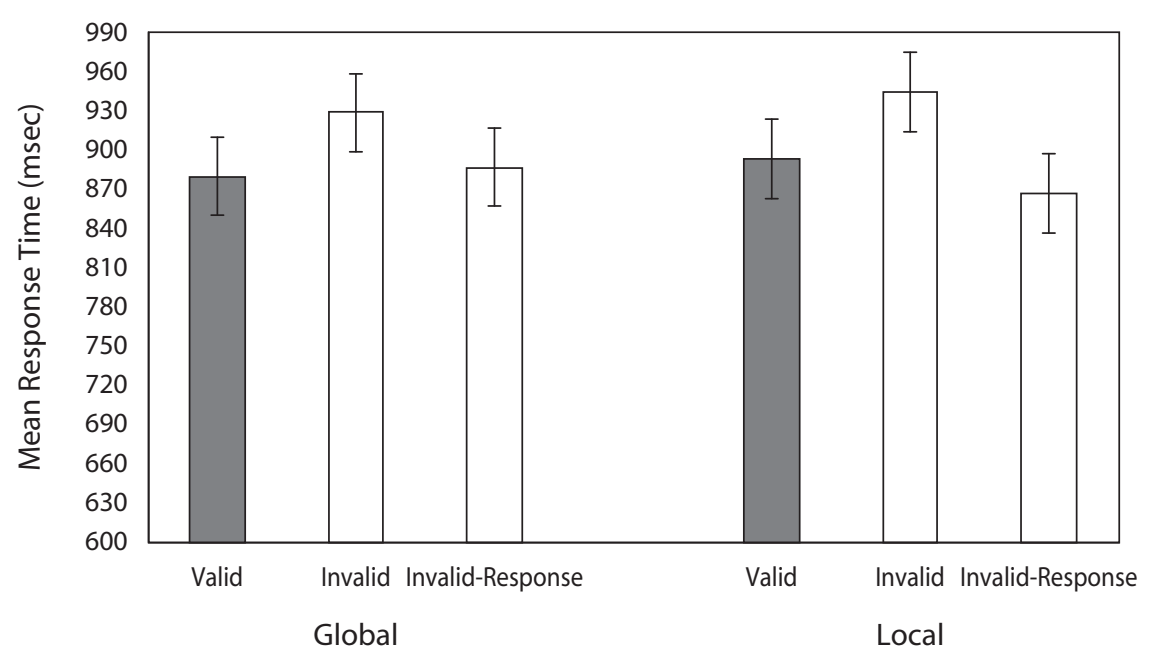

Figure 14. Experiment 2A: Effect of working memory on the responses to different levels of form as a function of the congruency of the local and global forms. Error bars represent standard errors of the means. 
Table 6

Error Rates in Experiment 2B

\begin{tabular}{|c|c|c|c|c|}
\hline Experiment & Level & Congruency & Validity & $\begin{array}{c}\text { Search } \\
\text { Errors }(\%)\end{array}$ \\
\hline \multirow[t]{18}{*}{ No memory } & \multirow[t]{9}{*}{ Global } & \multirow[t]{3}{*}{ Congruent } & Valid & 0.0 \\
\hline & & & Invalid-response & 0.2 \\
\hline & & & Neutral & 0.1 \\
\hline & & \multirow[t]{3}{*}{ Incongruent } & Valid & 0.0 \\
\hline & & & Invalid & 0.0 \\
\hline & & & Neutral & 0.4 \\
\hline & & \multirow[t]{3}{*}{ Control } & Valid & 0.1 \\
\hline & & & Invalid-response & 0.7 \\
\hline & & & Neutral & 0.0 \\
\hline & \multirow[t]{9}{*}{ Local } & \multirow[t]{3}{*}{ Congruent } & Valid & 0.1 \\
\hline & & & Invalid-response & 0.0 \\
\hline & & & Neutral & 1.1 \\
\hline & & \multirow[t]{3}{*}{ Incongruent } & Valid & 0.2 \\
\hline & & & Invalid & 0.2 \\
\hline & & & Neutral & 0.0 \\
\hline & & \multirow[t]{3}{*}{ Control } & Valid & 0.0 \\
\hline & & & Invalid-response & 0.1 \\
\hline & & & Neutral & 0.0 \\
\hline
\end{tabular}

participated for course credit or cash. All had normal or correctedto-normal vision.

Apparatus and Stimuli. The same apparatus was used as in the preceding experiments. All the stimuli (block letters and compound letters) had the same properties as those used previously.

Task and Procedure. Experiment 2B adopted the same experimental procedure as Experiment 2A. Trials were blocked into 14 parts, seven blocks each for the global and local levels. Each block of trials started with an English alphabetical letter (Courier New font, size 32). The letter was randomly chosen from among a group of seven (A, C, E, H, M, O, S). This letter was followed by the word global or local (the level to be attended to at the current block). The first letter was for participants to decide whether or not to do the search task in the ensuing presentations of stimuli: If the letter was the same as the following block letter (a prime in the present experiment, equivalent to the memory cue in Experiment 2A), participants were instructed to perform the hierarchical letter task at the level cued. If the letter was different from the following block letter, they were not to perform the task. This ensured that participants had to encode the letter cue but not hold it in memory. The importance of accuracy and the speed of response were equally emphasized.

\section{Results}

Errors in the search task were very low, at 3.2\% (see Table 6). There was no evidence of a speed-accuracy trade-off, and the data were not analyzed further, as in Experiment 2A. Four separate ANOVAs were conducted to test the overall global-local congruency effect following a neutral cue, and to test the effect of cue-target validity under each of the three congruency conditions (with congruent, incongruent, and control letters).

Congruency effect. We conducted a $2 \times 2$ ANOVA, with the factor being target level (global and local) and global-local congruency (congruent and incongruent stimuli, following a neutral cue). A strong global-local congruency effect was found when an ANOVA was conducted following neutral cues $[F(1,24)=52, p<.0001]$. There was also an effect of target level: Global targets were detected faster than local targets $[F(1,24)=5.4, p<$ $.03]$, but there was no target level $\times$ global-local congruency interaction $[F(1,24)=0.002, p=.97]$. Congruent trials were faster than incongruent trials, but this did not differ across local and global items. Mean RTs are given in Figure 15.

Validity effects under each congruency condition. Figure 16 gives the mean RTs for each condition. For each congruency condition a 2 (target level) $\times 3$ (cue-target validity) ANOVA was conducted.

Congruent displays. There was an effect of target level $[F(1,24)=27.3, p<.0001]$ but not of cue-target validity $[F(2,48)=1.2, p=.29]$, and there was no interaction $(F<1)$.

Incongruent displays. The effect of target level was reliable $[F(1,24)=6.44, p=.018]$. There was also an effect of cue-target validity $[F(2,48)=4.5, p=.016]$, but no interaction $(F<1)$

Control displays. The effect of target level was significant $[F(1,24)=11.5, p=.002]$. There was a borderline

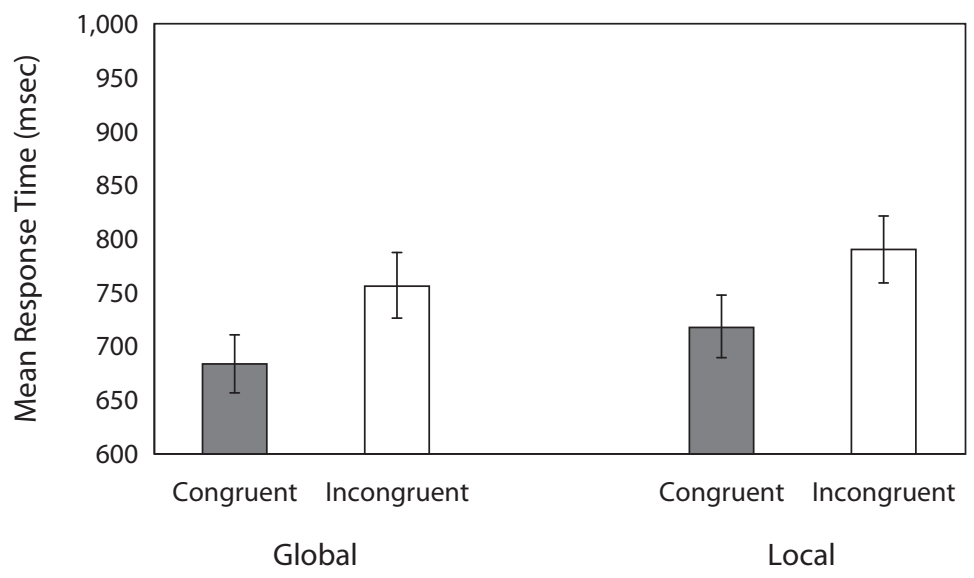

Figure 15. Experiment 2B: Overall congruency effect at each level of form. Error bars represent standard errors of the means. 


\section{Congruent}

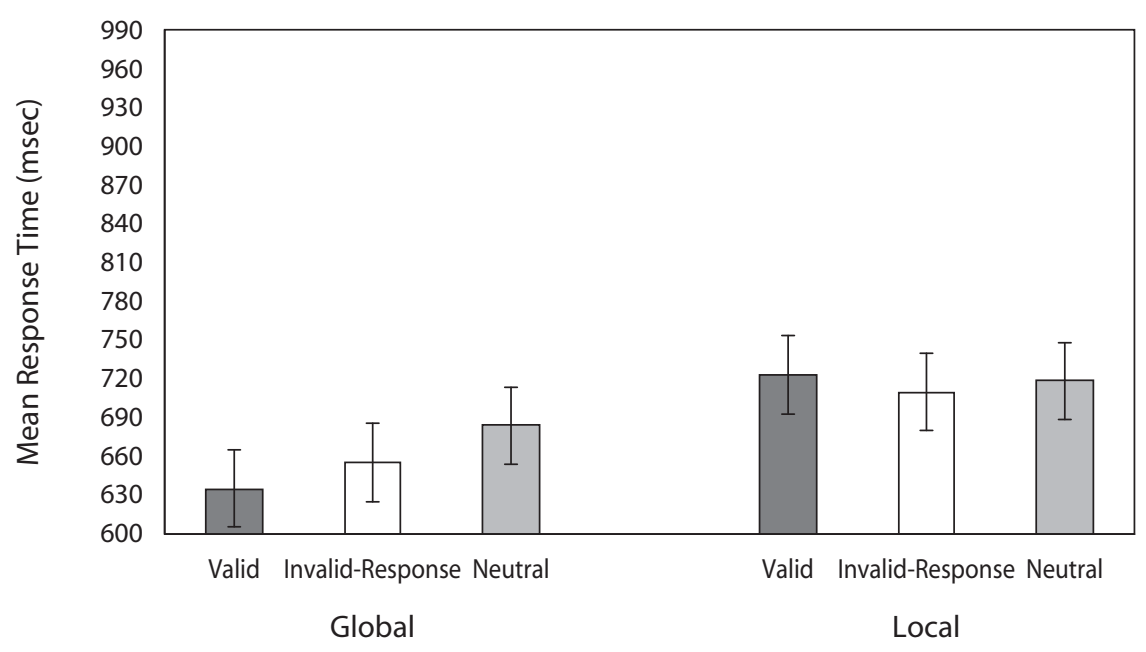

Incongruent

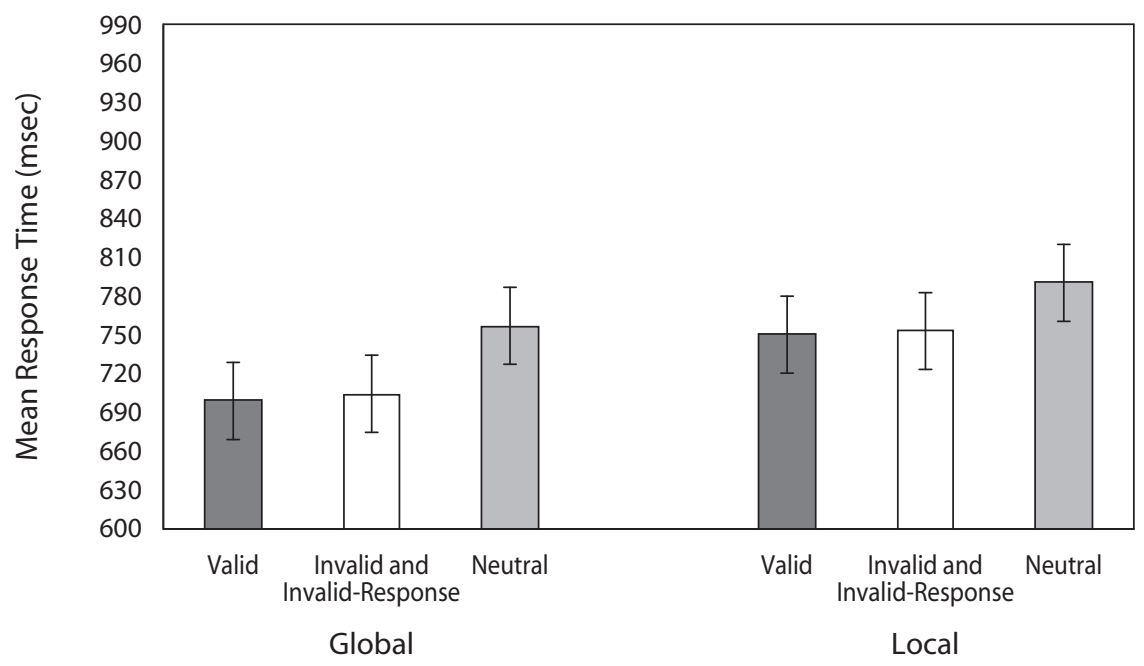

Control

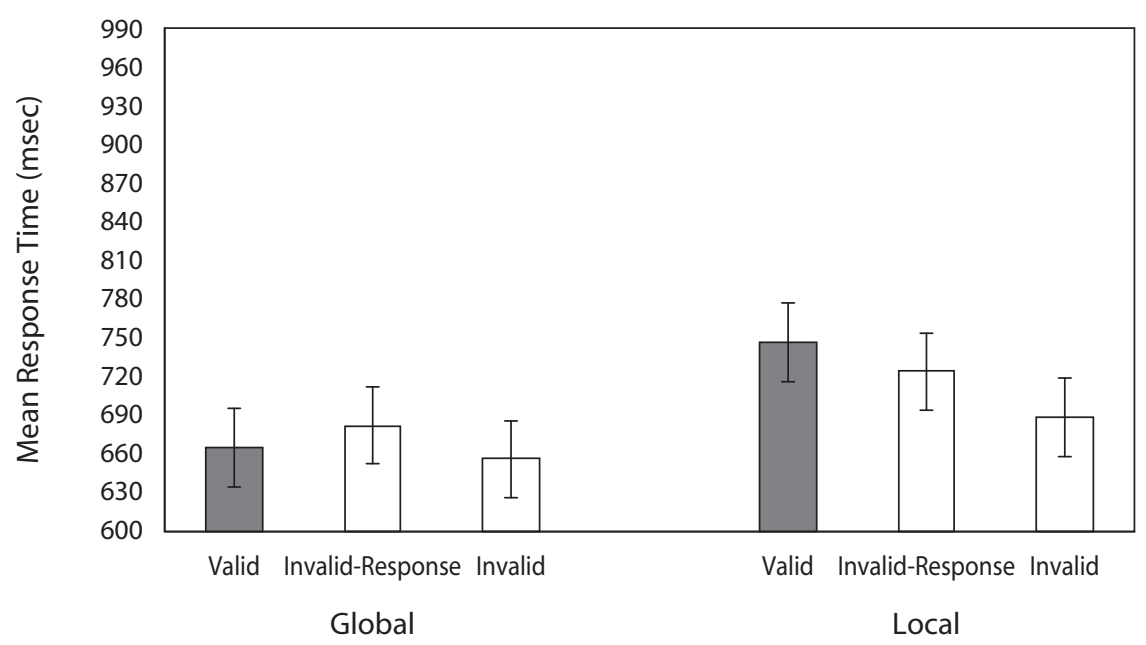

Figure 16. Experiment 2B: Effect of no memory priming by congruency on target perception at different levels. Error bars represent standard errors of the means. 
effect of cue-target validity $[F(2,48)=3.1, p=.053]$ but no interactions between target level and cue-target validity for any of the global-local congruency conditions $(F<1)$.

For incongruent stimuli, pairwise comparisons showed that there was a significant advantage for valid over neutral trials $[t(24)=-2.5, p=.02]$ and invalid and invalid-response trials over neutral trials $[t(24)=-2.8$, $p=.01]$. No difference was found between the trials with valid stimuli and with invalid and invalid-response stimuli $[t(24)=-0.2, p=.83]$.

\section{Discussion}

The results indicate two main points. First, the effects of the cue tended to be relatively small when participants performed the task in a focused rather than in a distributed mode of attention. For instance, in Experiment 1B there was a difference of around $100 \mathrm{msec}$ to global targets in the valid and neutral cue conditions. Here the greatest difference was around $30 \mathrm{msec}$, for stimuli with incongruent global and local forms. Second, when effects of validity occurred, they reflected benefits from the reappearance of the cue (compared with the neutral baseline), and this held, whether the cue was valid or invalid (matching the distractor rather than the target, with incongruent stimuli). This benefit, even on invalid trials, matches that found in Experiment 1B. Indeed, a cross-experiment comparison of trials for incongruent stimuli in Experiment 2A (WM) and Experiment $2 \mathrm{~B}$ (priming) with invalid and invalidresponse stimuli and neutral stimuli indicated an interaction between validity and experiment $[F(1,48)=5.2, p<$ $.05]$. There was a cost in the invalid and invalid-response condition in Experiment 2A (mean, $13 \mathrm{msec}$ ), but a benefit in Experiment 2B (mean, $45 \mathrm{msec}$ ). These results suggest that, when the cue had to be identified but not held in memory (Experiment 2B), there was a priming effect that facilitated identification of a target whenever the prime reappeared in the target letter. This occurred even though the prime in the invalid and invalid-response condition could activate a response competitor to the target and could cue attention to the nontarget level. ${ }^{2}$

A final point to note here is that a robust global advantage was present in this experiment across all the priming conditions. A similar trend was present in Experiment 1B, when the primes were identified but not held in memory, and in Experiment $1 \mathrm{C}$, when primes were held in memory but were never valid. On the other hand, there were no reliable differences between global and local levels in the baseline conditions of Experiments $1 \mathrm{~A}$ and 2A. One difference between Experiments $1 \mathrm{~A}$ and $2 \mathrm{~A}$ and the other experiments is that participants held a cue in WM in Experiments $1 \mathrm{~A}$ and $2 \mathrm{~A}$. This suggests that holding a prime in memory may weaken the global advantage.

\section{EXPERIMENT 3}

\section{Testing a Memory Refresh Account}

In Experiments 1 and 2, we provided evidence for the effects of WM differing from those of perceptual priming on the identification of hierarchical forms. The data suggest that top-down cuing of attention from WM operates differently from bottom-up perceptual priming. However, as we have noted, an alternative account of the WM results is that participants deliberately attend to matching information in the hierarchical letter, and this influences their responses to the hierarchical target. This memory refresh account was tested in Experiment 3. In this experiment, participants were asked to remember the size of an initial stimulus, rather than its identity. Memorytest events always maintained the same letter identity, and this letter was either the same size as the initial cue or a different size. Under these conditions, there is no incen-

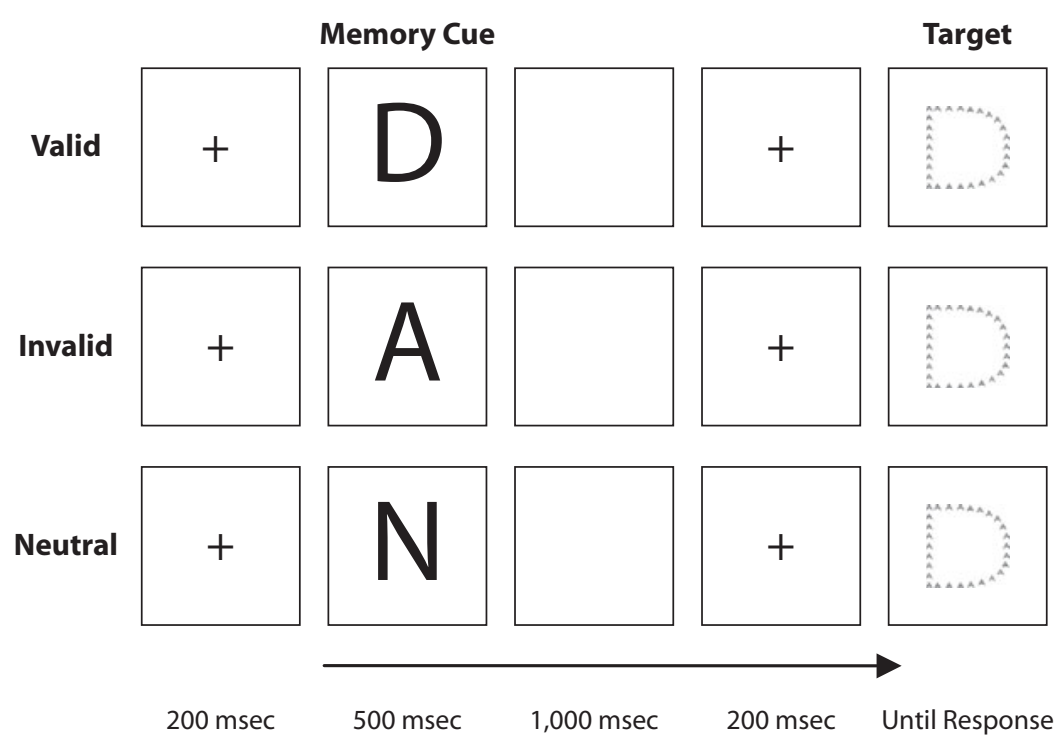

Figure 17. Examples of valid, invalid, and neutral conditions in Experiment 3. In these examples, the size of memory cues matched the global level of the hierarchical stimulus. 
Table 7

Error Data on Target-Present Trials in Experiment 3

\begin{tabular}{cclcc}
\hline $\begin{array}{c}\text { Target } \\
\text { Level }\end{array}$ & $\begin{array}{c}\text { Memory } \\
\text { of Size }\end{array}$ & Validity & $\begin{array}{c}\text { Search } \\
\text { Errors (\%) }\end{array}$ & $\begin{array}{c}\text { Memory } \\
\text { Errors (\%) }\end{array}$ \\
\hline \multirow{2}{*}{ Global } & \multirow{2}{*}{ Global } & Valid & 0.0 & 0.0 \\
& & Invalid & 0.2 & 0.2 \\
& \multirow{6}{*}{ Local } & Neutral & 0.2 & 0.1 \\
& & Valid & 0.5 & 0.2 \\
& & Invalid & 0.4 & 0.3 \\
& \multirow{2}{*}{ Global } & Neutral & 0.6 & 0.3 \\
& & Valid & 0.5 & 0.2 \\
& \multirow{2}{*}{ Local } & Invalid & 0.4 & 0.2 \\
& & Neutral & 0.6 & 0.2 \\
& & Valid & 0.3 & 0.3 \\
& & Invalid & 0.6 & 0.3 \\
& & Neutral & 0.3 & 0.2 \\
\hline
\end{tabular}

tive to attend to the identity of the cue in the hierarchical stimulus (refreshing memory for the identity would not help participants decide whether the letter was the same or a different size). We ask whether responses to the hierarchical target letter are still affected by re-presenting the identity of the WM stimulus in the target display. Soto and Humphreys (2009) found that responses to a target display could be affected by irrelevant as well as relevant properties of a stimulus held in WM. We tested whether task-irrelevant identity information from the WM cue affected responses based on a matching identity in the hierarchical stimulus.

\section{Method}

Participants. Thirty students and staff (age range, 19-27 years) at the School of Psychology of the University of Birmingham participated for course credit or cash. All had normal or corrected-to-normal vision.

Apparatus and Stimuli. The same apparatus was used as for Experiments 1 and 2.

'Iask and Procedure. Figure 17 illustrates the experimental conditions and sequence used in the present study. Each trial started with a block English alphabetical letter-A, N, or D—all of which were presented in equal frequency in two different sizes: The letter was either the size of the global level of the compound stimulus or the size of the letter representing the local level. There were 6 experimental conditions: 3 validity (of identity) conditions $\times 2$ size match conditions. The validity conditions were as follows: valid, when the identity of the memory cue matched that of the target item; invalid, when the identity of the memory cue was different from the target and represented the stimulus at the other nontarget level; and neutral, when the identity of the memory cue was different from the target and was not present at either of the levels. Each of these validity conditions had two size match conditions, in which the size of the memory cue was either the same as or different from the size of the target letter as represented at the level shown. The target was always the letter $\mathrm{D}$, matching the divided attention condition used in Experiment 1. Participants were instructed to remember the size of the memory cue, then to look for the target letter in the compound stimulus without regard to the level at which it was shown. On memory probe trials, the participants were asked to decide whether the size of the item presented for probe was the same as or different from the size of the item they had held in their memory. The identity of the memory probe item was kept constant on memory test trials, in order to discourage participants from memorizing the identity of the cue. There were four blocks of 120 trials, half of which were target-absent. For each block there were 10 trials for each condition.

\section{Results}

Target-present data. The error rate for the globallocal task was $4.6 \%$, and $2.5 \%$ for the memory test (see Table 7 for overall data summary). There was no evidence of a speed-accuracy trade-off, and the accuracy data were not analyzed further. A $2 \times 2 \times 3$ ANOVA was carried out on the mean RT data, with the factors of cue size (globalletter or local-letter size), target level (global or local), and validity (valid, invalid, or neutral, depending on the identity of the cue). The results are shown in Figure 18. There was a main effect of target level $[F(1,29)=9.5, p=$ $.004]$ but no effects of cue size $[F(1,29)=1.02, p=.32]$ or validity $[F(2,58)=2.0, p=.15]$. Two-way and threeway interactions were all nonsignificant.

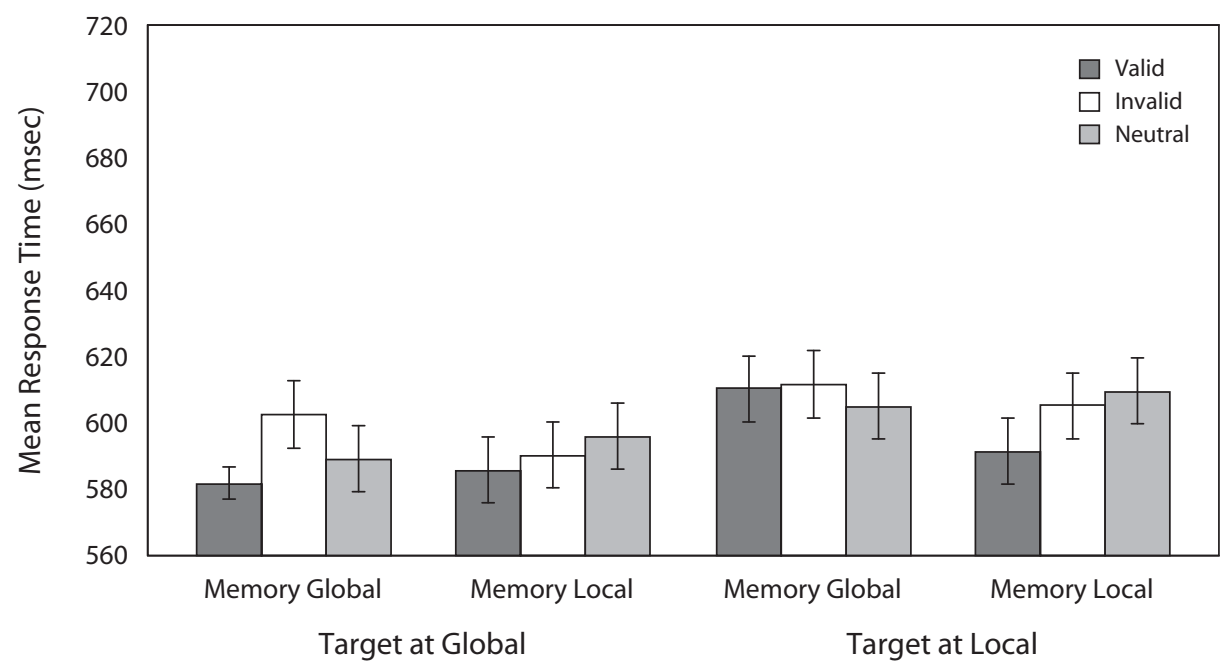

Figure 18. Effects of cue size and identity on the detection of targets in the hierarchical stimulus. Error bars represent standard errors of the means. 
Table 8

Error Data on Target-Absent Trials in Experiment 3

\begin{tabular}{clcc}
\hline $\begin{array}{c}\text { Memory } \\
\text { of Size }\end{array}$ & Validity & $\begin{array}{c}\text { Search } \\
\text { Errors (\%) }\end{array}$ & $\begin{array}{c}\text { Memory } \\
\text { Errors (\%) }\end{array}$ \\
\hline \multirow{2}{*}{ Global } & Valid (global) & 0.1 & 0.1 \\
& Valid (local) & 0.2 & 0.3 \\
& Neutral & 0.4 & 0.2 \\
Local & Valid (global) & 0.3 & 0.3 \\
& Valid (local) & 0.4 & 0.5 \\
& Neutral & 0.8 & 0.3 \\
\hline
\end{tabular}

Target-absent data. Again, there was no indication of a speed-accuracy trade-off. The error rates for the task and memory probe were $2.2 \%$ and $1.7 \%$, respectively (see Table 8 for the data summary). The data from target-absent trials were sorted into six conditions. There were two conditions of cue size (global-letter or local-letter), each of which had three cue-target validity conditions (valid, with the identity of the memory cue matching the target letter at the global level; valid, with the identity of the cue matching the letter at the local level; and neutral, with the identity of the memory cue not matching the letter at either of the two levels). The data were analyzed in a $2 \times 3$ ANOVA, with size of the cue (global and local sizes) and three levels of validity (valid global, valid local, and neutral) as factors. The results are shown in Figure 19. There was a strong main effect of cue-target validity $[F(2,58)=28.4, p<.0001]$ but no effect of cue size $[F(1,29)=2.57, p=.12]$. Cue size and cue-target validity did not interact $[F(2,58)=2.0$, $p=.14]$. Averaged across the two cue sizes, there were reliable differences between the valid global and neutral trials $[t(29)=5.7, p<.0001]$ and between the valid local and neutral trials $[t(29)=6.3, p<.0001]$. The valid global and valid local trials did not differ $[t(29)=0.06, p=.95]$.

\section{Discussion}

The data indicate effects of the identity but not of the size of the stimulus held in WM. As in Experiment 1A, there was a cost on absent trials when the identity of the cue matched one of the letters in the hierarchical stimulus. This result is consistent with an identity match cuing attention to the congruent level of the hierarchical stimulus, making it difficult to respond that the target is absent. The result suggests that, as in Soto and Humphreys (2009), there can be effects from irrelevant as well as relevant properties of stimuli held in WM. Indeed, there were no effects of the WM cue matching the size of one level of the hierarchical stimulus, either because size information does not cue attention, or because the size differences assessed on memory test trials were sufficiently large to allow size to be memorized only coarsely. Irrespective of this, the important result is that participants should have voluntarily attended to the size of stimuli in the hierarchical letter, not the identity, if attention was allocated in order to boost WM. Note that the identity of the cue remained the same on memory match and mismatch trials, so refreshing the identity of the memory cue would not benefit memory performance. Despite this, there was an effect of the identity of the cue. This goes against a memory refresh account of the WM effects.

\section{GENERAL DISCUSSION}

We have reported the first-ever study on the effects of irrelevant information in WM on the selection of hierarchical forms. Several critical results were established.

1. A cue held in WM strongly affected selection of hierarchical forms in a distributed attention task, when participants searched for a target that could appear at either

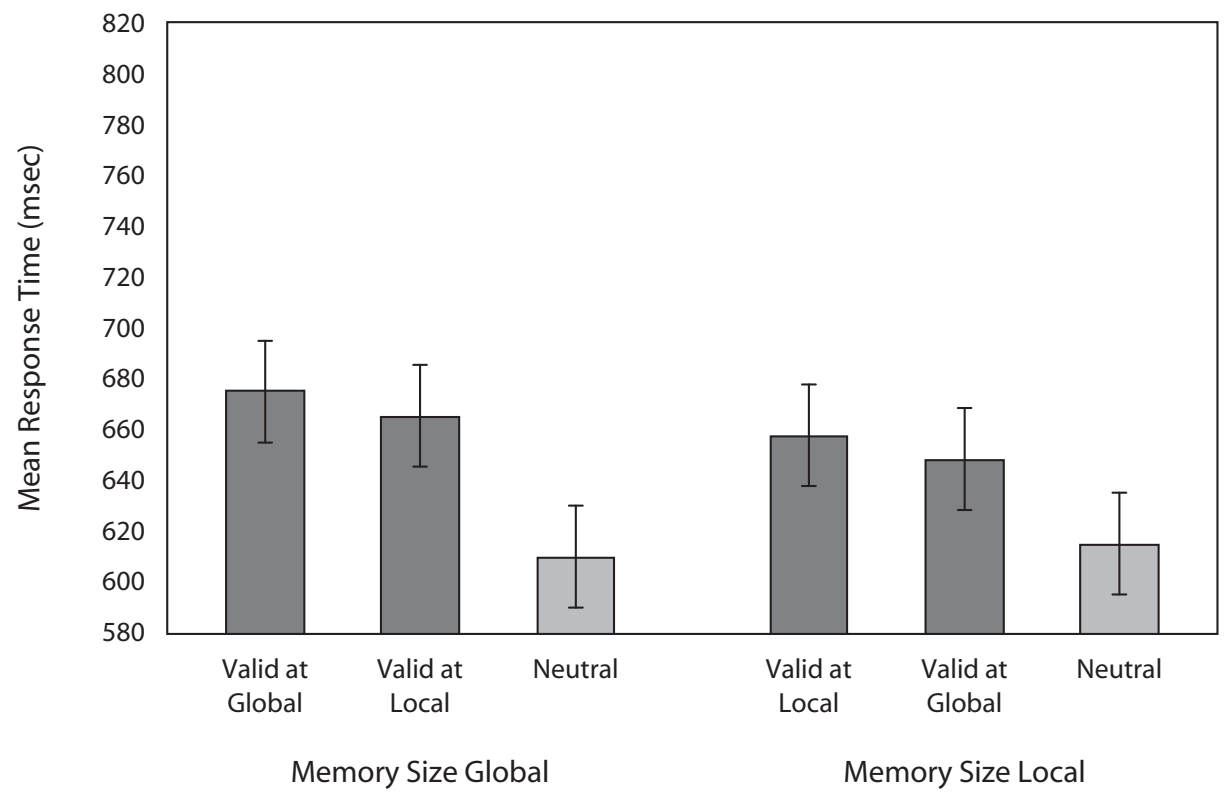

Figure 19. Target-absent data in Experiment 3: Effects of the size and identity of the memory cue. Error bars represent standard errors of the means. 
level of the hierarchical letter (Experiments 1A and 1C). Relative to when the cue did not appear in the hierarchical letter (the neutral baseline), RTs were facilitated when the cue was valid and disrupted when the cue was invalid.

2. The WM cue had its strongest effect when it matched the global level of the hierarchical letters (positive effects of valid cuing were larger, as were costs from invalid cuing: Experiment 1A).

3. The costs from invalid cuing occurred even when the WM cue was never valid, indicating that the effects arose automatically (Experiment 1C); and the effect also arose when participants could adopt a focused mode of attention (Experiment 2A).

4. In contrast to the effects when the cue was held in WM, there were only benefits to performance when the cue was merely identified and it reappeared within the hierarchical letter (Experiments 1B and 2B). This occurred not only when the cue matched the target (on valid trials) but also when the cue matched the distractor letter (on invalid trials in Experiment 1B, and on trials with invalid and invalid-response stimuli in Experiment 2B). There are opposite effects on performance from priming and from holding an item in WM.

5. The WM effects were confirmed under conditions in which it was not beneficial for participants to attend to information reappearing in the hierarchical stimulus, contrary to a memory refresh account of the data (Experiment 3).

Prior studies have shown that a cue held in WM can automatically affect the guidance of spatial attention to a search display (Downing, 2000; Olivers, Meijer, \& Theeuwes, 2006; Soto et al., 2005; Soto et al. 2006; Soto \& Humphreys, 2007). In these studies, the search target has often been more local than the WM item, when the memory item reappeared in the search display (e.g., a target appearing within a shape also held in WM). The present results indicate that this may be an important factor, since we found that WM-based guidance of attention was strongly tied to selection at a global level. The present results go beyond previous studies of WM effects, then, by indicating that there is not only spatial guidance of selection (cf. Soto et al., 2005), but also guidance to select visual stimuli at different levels of hierarchical representation, even when stimuli fall at the same location (e.g., local elements falling within a global form). This guidance of attention from WM is stronger to global levels of form.

The present results showed costs to selection when the WM cue matched a distractor at the nontarget level. One account of these costs is that attention is directed by an invalid cue to the wrong level of the hierarchical form, and this slows selection of the target at the other level. An alternative proposal is that the WM cue is suppressed, and this slows processing of the hierarchical target when the cue is represented in that stimulus. However, it is difficult to see why this suppression process would not affect valid as well as invalid trials, yet performance was facilitated on valid trials. This proposal also does not fit with the existing neuroimaging data on WM effects on attention, where the WM condition increases activation for re-presented stimuli and suppression is shown only under priming conditions (Soto et al., 2007). As we discuss below, these imaging data do fit with our results in the identification conditions (Experiments 1B and 2B). We conclude that the costs on invalid trials were due to the WM cue directing attention to the wrong level of the stimulus, slowing target detection at the other level.

\section{Distributed Attention Versus a Wide Attentional Window}

As noted at the beginning of this article, Hernández et al. (2010) have reported that effects of the WM cue were strong when participants adopted a wide attentional window (see also Belopolsky, Zwaan, Theeuwes, \& Kramer, 2007, for similar effects with bottom-up cuing of attention). In contrast to this, the magnitude of the validity effect in the present study was the same under distributed and focused attention conditions (Experiments 1A and 2A). In Experiment 2, participants could preselect a particular spatial frequency to attend to, but cuing effects remained. This result fits with the idea that both high and low spatial frequency components of a display are extracted in parallel, and so either can be cued from WM, even if participants are trying to focus attention at one level. Selective attention may influence the "read-out" of particular spatial frequencies, but not the coding of different frequencies in the first place. In addition, we propose that focusing on a target spatial frequency is not the same as adopting a broad attentional window, and we assume that a broad attentional window is adopted here with focused as well as distributed attention (e.g., when a global item has to be selected). When a broad attentional window is adopted, the cue affects performance for focused and distributed attention conditions alike.

\section{Bottom-Up Perceptual Priming}

The data in the priming condition provide a striking contrast to those found under WM conditions. When the cue was held in memory, there were effects on attentional guidance. When the prime was merely identified, effects on attentional guidance were minimized (e.g., there was no cost on invalid trials), but effects reflected enhanced perceptual processing when cues were repeated at both target and nontarget levels. RTs were then facilitated on invalid relative to neutral trials. These results are consistent with the data on attentional guidance using functional brain imaging - in particular, the evidence for repetition suppression when participants simply identified the cue and the cue was then re-presented in the search array (Soto et al., 2007). Neural repetition suppression may reflect facilitated perceptual processing of stimuli, with less information being required for stimulus recognition when the system is primed. Here, priming of one of the letters making up the hierarchical form appears to facilitate processing of the whole form, even when the prime letter reappeared at the nontarget level (on invalid trials). However, when the cue is also held in WM, the neuroimaging results indicate top-down enhancement of cued representations when the stimulus is re-presented in the search display (Soto et al., 2007). This enhanced activity, driven by the WM representation, guides attention to the re-presented stimulus. This 
will disrupt responses when the cue is invalid, since the nontarget letter will be selected, and costs to performance result. One other result to note is that this bottom-up priming effect held both when participants were in a distributed attention mode and when they had a focused attentional set (in Experiments 1B and 2B). This contrasts with the effects of valid cuing of attention from WM, which was weakened in a focused attention mode (in Experiment 2A, compared with Experiment 1A). The effects of bottomup priming may arise irrespective of the perceptual set of participants.

\section{CONCLUSIONS}

We have shown that attention can be biased to a level of a hierarchical form when a stimulus in WM matches the identity of the letter at that level; this disrupts performance when WM cues attention to a distractor level. The effect is not due to participants' attending to matching information in the hierarchical stimulus in order to refresh their memory for the cue's identity. In contrast to the WM effects, priming a letter identity facilitates the perceptual processing of a compound letter without cuing attention. In this case, matches between a prime and a distractor level of the compound facilitate identification, whether the prime is valid or invalid in relation to the target. Although matches to WM cue attention, priming facilitates perceptual processing without necessarily biasing attention.

\section{AUTHOR NOTE}

The present experiments were supported by grants from the BBSRC and MRC, U.K. Correspondence concerning this article should be addressed to J.-I. Kim or G. W. Humphreys, School of Psychology, University of Birmingham, Birmingham B15 2TT, England (e-mail: j.kim.1@bham .ac.uk org.w.humphreys@bham.ac.uk).

\section{REFERENCES}

Anderson, G. M., Heinke, D., \& Humphreys, G. W. (in press). Featural guidance in conjunction search: The contrast between orientation and color. Journal of Experimental Psychology: Human Perception \& Performance.

Belopolsky, A. V., Zwann, L., Theeuwes, J., \& Kramer, A. F. (2007). The size of an attentional window modulates attentional capture by color singletons. Psychonomic Bulletin \& Review, 14, 934-938.

Desimone, R., \& Duncan, J. (1995). Neural mechanisms of selective visual attention. Annual Review of Neuroscience, 18, 193-222.

DownING, P. E. (2000). Interactions between visual working memory and selective attention. Psychological Science, 11, 467-473.

Duncan, J. (1998). Converging levels of analysis in the cognitive neuroscience of visual attention. Philosophical Transactions of the Royal Society B, 353, 1307-1317.

HAN, S. W., \& KIM, M.-S. (2009). Do the contents of working memory capture attention? Yes, but cognitive control matters. Journal of Experimental Psychology: Human Perception \& Performance, 35, 1292-1302.

Henson, R., Shallice, T., \& Dolan, R. (2000). Neuroimaging evidence for dissociable forms of repetition priming. Science, 287, 12691272 .

Hernández, M., Costa, A., \& Humphreys, G. W. (2010). The size of an attentional window affects working memory guidance. Attention, Perception, \& Psychophysics, 72, 963-972.

Hodsoll, J., \& Humphreys, G. W. (2001). Driving attention with the top down: The relative contribution of target templates to the linear separability effect in the size dimension. Perception \& Psychophysics, 63, $918-926$.
Huberle, E., \& Karnath, H.-O. (2006). Global shape recognition is modulated by the spatial distance of local elements-Evidence from simultanagnosia. Neuropsychologia, 44, 905-911.

HüBNER, R. (1993). Different ways of modeling spatial-frequency uncertainty in visual signal detection. Biological Cybernetics, 69, 457-462.

HüBNER, R. (1996). The efficiency of different cue types for reducing spatial-frequency uncertainty. Vision Research, 36, 401-408.

Hughes, H. C., Fendrich, R., \& Reuter-Lorenz, P. A. (1990). Global versus local processing in the absence of low spatial frequencies Journal of Cognitive Neuroscience, 2, 272-282.

Julesz, B., \& Papathomas, T. V. (1984). On spatial-frequency channels and attention. Perception \& Psychophysics, 36, 398-399.

KIMcHI, R. (1992). Primacy of wholistic processing and global/local paradigm: A critical review. Psychological Bulletin, 112, 24-38.

Kimchi, R., \& PAlmer, S. E. (1982). Form and texture in hierarchically constructed patterns. Journal of Experimental Psychology: Human Perception \& Performance, 8, 521-535.

Kinchla, R. A., \& Wolfe, J. M. (1979). The order of visual processing: "Top-down," "bottom-up," or "middle-out." Perception \& Psychophysics, 25, 225-231.

LAMb, M. R., \& Robertson, L. C. (1988). The processing of hierarchical stimuli: Effects of retinal locus, locational uncertainty, and stimulus identity. Perception \& Psychophysics, 44, 172-181.

Lux, S., Thimm, M., Marshall, J. C., \& Fink, G. R. (2006). Directed and divided attention during hierarchical processing in patients with visuo-spatial neglect and matched healthy volunteers. Neuropsychologia, 44, 436-444.

NAVON, D. (1977). Forest before trees: The precedence of global features in visual perception. Cognitive Psychology, 9, 353-383.

Olivers, C. N. L. (2009). What drives memory-driven attentional capture? The effects of memory type, display type, and search type. Journal of Experimental Psychology: Human Perception \& Performance, 35, 1275-1291.

Olivers, C. N. L., Meijer, F., \& Theeuwes, J. (2006). Feature-based memory-driven attentional capture: Visual working memory content affects visual attention. Journal of Experimental Psychology: Human Perception \& Performance, 32, 1243-1265.

PAshler, H., \& Shiu, L.-P. (1999). Do images involuntarily trigger search? A test of Pillsbury's hypothesis. Psychonomic Bulletin \& Review, 6, 445-448.

Shalev, L., Humphreys, G. W., \& Mevorach, C. (2005). Global processing of compound letters in a patient with Balint's syndrome. Cognitive Neuropsychology, 22, 737-751.

Shulman, G. L., Sullivan, M. A., Gish, K., \& SaKoda, W. J. (1986). The role of spatial-frequency channels in the perception of local and global structure. Perception, 15, 259-273.

Soto, D., Heinke, D., Humphreys, G. W., \& Blanco, M. J. (2005). Early, involuntary top-down guidance of attention from working memory. Journal of Experimental Psychology: Human Perception \& Performance, 31, 248-261.

Soto, D., Hodsall, J., Rotshtein, P., \& Humphreys, G. W. (2008). Automatic guidance of attention from working memory. Trends in Cognitive Sciences, 12, 342-348.

Soto, D., \& Humphreys, G. W. (2007). Automatic guidance of visual attention from verbal working memory. Journal of Experimental Psychology: Human Perception \& Performance, 33, 730-737.

Soto, D., \& Humphreys, G. W. (2009). Automatic selection of irrelevant object features through working memory: Evidence for top-down attentional capture. Experimental Psychology, 56, 165-172.

Soto, D., Humphreys, G. W., \& Heinke, D. (2006). Working memory can guide pop-out search. Vision Research, 46, 1010-1018.

Soto, D., Humphreys, G. W., \& Rotshtein, P. (2007). Dissociating the neural mechanisms of memory-based guidance of visual selection. Proceedings of the National Academy of Sciences, 104, 17186-17191.

Vuilleumier, P., Schwartz, S., Duhoux, S., Dolan, R. J., \& Driver, J. (2005). Selective attention modulates neural substrates of repetition priming and "implicit" visual memory: Suppressions and enhancements revealed by fMRI. Journal of Cognitive Neuroscience, 17, 1245-1260.

Wig, G. S., Buckner, R. L., \& Schacter, D. L. (2009). Repetition priming influences distinct brain systems: Evidence from task-evoked data and resting-state correlations. Journal of Neurophysiology, 101, 2632-2648. 
Wiggs, C. L., \& Martin, A. (1998). Properties and mechanisms of perceptual priming. Current Opinion in Neurobiology, 8, 227-233.

WoLFE, J. M. (2005). Guidance of visual search by preattentive information. In L. Itti, G. Rees, \& J. K. Tsotsos (Eds.), Neurobiology of attention (pp. 101-104). San Diego: Academic Press.

Woodman, G. F., \& Luck, S. J. (2007). Do the contents of visual working memory automatically influence attentional selection during visual search? Journal of Experimental Psychology: Human Perception \& Performance, 33, 363-377.

Yovel, G., Yovel, I., \& Levy, J. (2001). Hemispheric asymmetries for global and local visual perception: Effects of stimulus and task factors. Journal of Experimental Psychology: Human Perception \& Performance, 27, 1369-1385.

\section{NOTES}

1. For Experiment 2A, the data were taken from the control condition. Only in that condition was the nontarget level of the hierarchical letter irrelevant to the response (as was always the case in Experiment 1A). Also, the neutral condition from Experiment $1 \mathrm{~A}$ and the invalid-response conditions from Experiment 2A were omitted, since they had no equivalent in the other experiment.

2 . These data from Experiment 2 also help to counter one alternative account of the results from Experiment 1. The alternative account of Experiment 1 suggests that the contrast between the WM and priming conditions (in Experiments 1A and 1B) was due to slower RTs in the neutral baseline in the priming conditions [although a $2 \times 2$ ANOVA directly contrasting the two neutral conditions in Experiments $1 \mathrm{~A}$ and $1 \mathrm{~B}$ failed to reveal any differences; $F(1,48)=2.65, p=.11$ ], not to a difference between WM and priming. However, Experiment 2 showed a similar contrast between costs from invalid cues in WM and benefits from priming (e.g., with incongruent stimuli), but in this case baseline (neutral) RTs were faster in the priming than in the WM condition. This pattern in the baseline is opposite to that in Experiment 1. We conclude that nonsignificant shifts in the baselines were not critical here.

(Manuscript received August 7, 2009; revision accepted for publication April 1, 2010.) 